\title{
Effectiveness of interventions to directly support food and drink intake in people with dementia: systematic review and meta-analysis
}

Asmaa Abdelhamid ${ }^{1,9}$, Diane Bunn ${ }^{1}$, Maddie Copley², Vicky Cowap ${ }^{3}$, Angela Dickinson $^{4}$, Lucy Gray $^{1}$, Amanda Howe ${ }^{1}$, Anne Killett ${ }^{5}$, Jin Lee ${ }^{1}$, Francesca Li ${ }^{1}$, Fiona Poland ${ }^{5}$, John Potter ${ }^{1,6}$, Kate Richardson ${ }^{6}$, David Smithard ${ }^{7}$, Chris Fox ${ }^{1,8}$ and Lee Hooper ${ }^{1 *}$ (D)

\begin{abstract}
Background: Eating and drinking difficulties are recognised sources of ill health in people with dementia. In the EDWINA (Eating and Drinking Well IN dementiA) systematic review we aimed to assess effectiveness of interventions to directly improve, maintain or facilitate oral food and drink intake, nutrition and hydration status, in people with cognitive impairment or dementia (across all settings, levels of care and support, types and degrees of dementia). Interventions included oral nutrition supplementation, food modification, dysphagia management, eating assistance and supporting the social element of eating and drinking.

Methods: We comprehensively searched 13 databases for relevant intervention studies. The review was conducted with service user input in accordance with Cochrane Collaboration's guidelines. We duplicated assessment of inclusion, data extraction, and validity assessment, tabulating data, carrying out random effects meta-analysis and narrative synthesis.

Results: Forty-three controlled interventions were included, disappointingly none were judged at low risk of bias. Oral nutritional supplementation studies suggested small positive short term but unclear long term effects on nutritional status. Food modification or dysphagia management studies were smaller and of low quality, providing little evidence of an improved nutritional status. Eating assistance studies provided inconsistent evidence, but studies with a strong social element around eating/drinking, although small and of low quality provided consistent suggestion of improvements in aspects of quality of life. There were few data to address stakeholders' questions.

Conclusions: We found no definitive evidence on effectiveness, or lack of effectiveness, of specific interventions but studies were small and short term. People with cognitive impairment and their carers have to tackle eating problems despite this lack of evidence, so promising interventions are listed. The need remains for high quality trials tailored for people with cognitive impairment assessing robust outcomes.
\end{abstract}

Systematic review registration: The systematic review protocol was registered (CRD42014007611) and is published, with the full MEDLINE search strategy, on Prospero [1].

Keywords: Dementia, Aged, Eating, Drinking, Meta-analysis, Diet, Malnutrition, Dehydration

\footnotetext{
*Correspondence: I.hooper@uea.ac.uk

${ }^{1}$ Norwich Medical School, University of East Anglia, Norwich Research Park,

Norfolk NR4 7TJ, UK

Full list of author information is available at the end of the article
} 


\section{Background}

Over 47 million people are living with dementia worldwide [2], and age-standardized prevalence for people aged $\geq 60$ years is $5-7 \%[3,4]$. The incidence of dementia progressively increases with age, so that as populations become older prevalence rises, despite younger cohorts having a lower risk of dementia [5, 6]. As their disease progresses, the needs of people with dementia become increasingly complex: $76 \%$ of institutionalised people with dementia needed to be fed, refused food or choked on liquid or solid foods [7]. Eating and drinking difficulties are a major source of ill health and stress for people living with dementia and their carers, and addressing these difficulties was identified as a top-ten research priority by people with dementia and their formal and informal carers [8], as well as an international survey of experts [9]. People with dementia are more likely to drink insufficient fluid, be malnourished, and malnutrition risk increases as dementia progresses [10-13]. While hospital admissions for dehydration and anorexia or malnutrition account for $1 \%$ and $3 \%$ of total hospital admissions, persons with dementia account for ten times more admissions when compared to age-matched controls [14]. Reasons may include poor appetite, decreased sense of thirst, increased fluid losses related to reduced urinary concentrating capacity, forgetting to eat and drink, days less structured around food and drink, reduced social contact, physical difficulties in shopping or food preparation, changes in food preferences, lack of formal or informal carer training and problems with chewing and swallowing [15-17]. The nature of difficulties vary with the stage of the illness.

This systematic review addresses these research priorities around eating and drinking in dementia by systematically reviewing existing research on direct and indirect interventions aiming to improve, maintain or facilitate the food or drink intake in adults with dementia at any age, at any stage (including mild cognitive impairment, $\mathrm{MCI}$ ), and in any setting. Direct interventions aimed to modify food and/or drink, provide food or drink-based supplements, provide social support, assist with eating or drinking or manage swallowing problems, alone or as part of multicomponent interventions. Indirect interventions included dining environment or food service modifications, educational, behavioral, exercise-type and multicomponent interventions. This paper reports on direct interventions, whilst the companion publication reports on indirect interventions [18]. No previous systematic review has addressed the full scope of interventions in people with this range of cognitive impairment. Key objectives included summarising evidence of effectiveness of interventions rigorously to minimise bias, addressing questions raised by our lay stakeholders (we involved stakeholders in designing the review to ensure we addressed questions relevant for people with dementia and their carers) and highlighting research priorities $[8,9,19]$.

\section{Methods}

We developed the systematic review protocol collaboratively, and the review team included lay stakeholders, subject experts and methodological experts. Lay stakeholders included members from AgeUK Norfolk and NorseCare (residential homes group). We worked with two patient and public involvement groups (the Public \& Patient Involvement in Research, PPIRes, from Norfolk and Suffolk and the Public Involvement in Research Group, PIRG, from the University of Hertfordshire) to develop additional specific questions for the review. The protocol is published, with the full MEDLINE search strategy, on Prospero [1]. The review was conducted in accordance with Cochrane Collaboration's guidelines [20], and reported in accordance with PRISMA guidance [21]. Study methods and specific questions posed by lay stakeholders are reported in full (Additional file 1), and summarised below.

\section{Criteria for inclusion}

We included randomised (RCTs) and non-randomised (CCTs) intervention studies that fulfilled the following criteria:

- Participants: $\geq 3$ adults diagnosed with any type/stage of dementia or mild cognitive impairment $(\mathrm{MCI})$ or where the mean Mini Mental State Examination (MMSE) score plus one standard deviation was $\leq 26$, in any setting.

- Duration: $\geq 5$ consecutive days.

- Interventions: aimed to modify food and/or drink, provide food- or drink-based supplements, assist with eating or drinking or manage swallowing problems (pharmacological and pill-based supplements were excluded).

- Outcomes: nutrition or hydration status [22]; quantity, quality or adequacy of food or fluid intake, ability to eat independently, swallow without aspirating, enjoyment of food or drink or meaningful activity (activity around food or drink that is personally fulfilling, that people enjoy, look forward to or find important). Note - studies were only included if they collected at least one of these outcomes, but where studies were included we also extracted, and report, data provided on the following outcomes: quality of life, functional or cognitive status, views or attitudes, cost effectiveness, resource use, mortality and health outcomes. 


\section{Search strategy}

We developed a complex MEDLINE search strategy and adapted it for 12 further databases (EMBASE, CINAHL, PsychInfo, five Cochrane Databases, meta-register of controlled trials, ALOIS (Cochrane Dementia and Cognitive Improvement Group comprehensive register of dementia trials), Dissertation and Thesis abstracts, and International Alzheimer's Disease Research Portfolio (IADRP). Bibliographies of included studies and lists of included/excluded studies from relevant reviews were checked [23-28]. There were no language or date limitations.

\section{Study selection and data collection}

Inclusion was assessed by two reviewers independently. Titles and abstracts were screened and full-text papers obtained if either reviewer considered it potentially eligible; full text papers were grouped into studies, and the review assessed interventions (some studies tested more than one intervention, while some interventions were described in several published papers) and assessed for inclusion. Corresponding authors were contacted where papers were published in languages other than English or there were insufficient data to assess suitability for inclusion or outcomes.

Data (publication details, participants, intervention, comparison, outcomes as above plus quality of life, functional or cognitive status, views or attitudes, cost effectiveness, resource use, mortality, health outcomes) and quality characteristics were extracted independently by two reviewers, discrepancies were resolved through discussion. Methodological quality was assessed using Cochrane risk of bias tool $[20,29]$. In addition to generic criteria, we assessed funding bias, validity of dementia diagnosis, outcome measures and baseline comparability between groups. We considered a study at low risk of bias where it was at low risk of both selection biases (was randomised and had appropriate allocation concealment) and detection bias (blinding of outcome assessment).

\section{Data synthesis}

Studies were grouped by type of intervention then study design (RCT/ CCT) for tables and narrative synthesis. CCTs included non-randomised studies with a concurrent comparator group and before-after (prepost) studies, collectively referred to as CCTs. Randomeffects meta-analysis of RCTs using Review Manager (RevMan 5.3) software was conducted where studies were suitably comparable (CCTs were summarised narratively). Heterogeneity was quantified using $\mathrm{I}^{2}$ [20]. As interventions are most useful if they have a lasting effect, subgrouping was by time from inception of the intervention to assessment of effects $(\leq 12$ weeks, $>12$ 26 weeks, $>26$ weeks).
Secondary analyses used subgrouping to address additional questions formulated by lay stakeholders (Table 1), and an additional question raised while conducting the review, on whether interventions that focussed on improving social contact of people with dementia, in the context of food and drink, were successful in supporting review outcomes.

\section{Results}

Searches identified 15468 citations (references and bibliographies added 37). After de-duplication we assessed 13863 titles and abstracts, and collected 293 full text papers for further assessment. Forty three direct interventions were included (Fig. 1).

Included interventions were from Europe (22 interventions), North America (17), Europe and the USA (multi-national study, one intervention, Brazil, Taiwan and New Zealand (one each). Most interventions (36) were conducted in institution or hospital settings, with four in day centres or the community, and unclear setting in one. Twenty eight interventions stated that participants were diagnosed with dementia, three with $\mathrm{MCI}$, and in 12 cognitive impairment was assumed from cognitive scores or setting. Dementia staging was possible in 19 interventions, of which four were severe, two moderate, three mild dementia, three $\mathrm{MCI}$, two moderate to severe, two mild to moderate and three mixed. Type of dementia was not always specified, but 13 interventions included participants with Alzheimer type dementia (AD) and eight included people with various dementia types.

Direct interventions were categorised into: oral supplements, food/drink modification, swallowing problems management, eating assistance and social support. Findings are presented under these headings below. Study characteristics and results are summarised in Tables 2 and 3, for full details of included studies see Additional file 2.

\section{Oral nutrition supplement (ONS) interventions Effects of ONS}

Twenty seven interventions (including approximately 1300 participants) [30-55] investigated effects of ONS. We defined these as studies where any oral food or drink was offered in addition to standard provision (including commercial ONS preparations and additional snacks/drinks). In 19 interventions the supplement was the only intervention and compared to usual care or placebo, whilst in two it was part of a multicomponent intervention [31,33] and in four was compared to another intervention $[48,50,54,55]$. Twenty studies were RCTs and six, CCTs. Study duration was from three to 80 weeks.

Few studies demonstrated low risk of selection bias, performance bias, detection bias or funding bias, and no studies were at low risk of bias overall (Additional file 3: Figure S3.1). Risk of attrition bias was low in over half of 
Table 1 Specific review questions formulated by members of the lay stakeholders, and the evidence found to address these questions

\begin{tabular}{ll}
\hline Area & Questions from lay stakeholders \\
\hline Type of dementia & For people with different types of \\
& dementia (Alzheimer's, vascular, \\
& dementia with Lewy bodies, other \\
& types or mixed types), what \\
interventions can help to maintain \\
or improve food intake or nutritional \\
status and fluid intake or hydration status?
\end{tabular}

Stage of dementia

Setting

Emotional and social issues

Meaningful Activity

Individualised interventions
What interventions can help to maintain or improve food intake or nutritional status and fluid intake or hydration status in people with mild cognitive impairment, mild/moderate/severe dementia?
1. For people with dementia living in residential care or residing in a medical setting, what interventions can help to maintain or improve food intake or nutritional status and fluid intake or hydration status?

2. For people with dementia living in their own homes with or without a carer (full-time or occasional; close relative or paid carer), what interventions can help to maintain or improve food intake or nutritional status and fluid intake or hydration status?

For people with dementia, does emotional closeness of the carer (e.g. close relative vs paid carer) affect the outcomes?

1. For people with dementia, what interventions aimed at improving or maintaining food and/or fluid intake, nutrition or hydration status, support meaningful activity (activity around food or drink that is personally fulfilling, that people enjoy, look forward to or find important)?

2. For people with dementia, are there any interventions that decrease food or fluid intake, diminish enjoyment or quality of life, or diminish meaningful activity or social inclusion?

Do individualised interventions appear more effective than those that are not individualised, in helping people with dementia to maintain or improve food and/or drink intake, nutrition or hydration status (or related outcomes)?

1. Do interventions to assess swallowing (and where necessary treat swallowing problems) have any effect on food or drink intake, nutrition

Review findings

Less than half of studies indicated type of dementia of participants, but most that did enrolled people with AD. Results of 8 ONS studies including $A D$ patients were not consistent - some studies reported improvement in nutritional status or intake, others no effect. Studies of other interventions were too few to compare or inform conclusions.

Less than half of the studies had any data on stage of dementia of participants. Potential interventions are shown in Table 4, but in studies of people with mild, moderate and/or severe dementia oral nutritional supplements (ONS) improved one or more markers of nutritional status (though usually not all markers, and only over short periods of time). Three studies of fruit juice supplements in people with mild cognitive impairment showed little effect. Shared mealtimes with staff and a commercial lyophilised food also appeared to improve some markers of nutritional status in people with severe dementia, while social interventions (supporting social interactions around food and drink) appeared to improve measures of participation, interactions, happiness, autonomy and involvement in people with mild, moderate and severe dementia.

Most of the studies were conducted in various residential or nursing settings, and very few in participants own homes. Generally, effectiveness of interventions related to the effectiveness of interventions in residential settings.

Emotional closeness to the carer was not ever reported in studies, and carers generally appeared to be professional rather than family carers.

Few studies measured quality of life or happiness using a validated scale. However, some studies especially those with a strong social element (see main review) reported improved autonomy, involvement and interest of participants. Few interventions reported diminished intake or any poorer outcomes, except for a study that gave supplemental yogurt at breakfast, which resulted in reduced weight (possibly as the result of replacing rather than supplementing usual food)

Studies of ONS did not offer individualised interventions (based on needs and preferences of participants) beyond a choice of flavours, but the one study of individualised snacks did not suggest they were helpful. Multicomponent individualised interventions were more positive, suggesting useful effects on some nutritional outcomes. Individualised dysphagia diet and a multicomponent food modification diet appeared to improve weight, and individualised eating assistance was not clearly helpful.

Studies assessing interventions for swallowing problems were generally inconclusive except that individual and multicomponent interventions 
Table 1 Specific review questions formulated by members of the lay stakeholders, and the evidence found to address these questions (Continued)

or hydration status (or related outcomes)?

2. Do interventions to improve oral hygiene have

any effect on food or drink intake, nutrition or

hydration status (or related outcomes)?

Interventions in

acute illness
Are there any interventions that are particularly effective in helping people with dementia to maintain or improve food and/or drink intake, nutrition or hydration status (or related outcomes) during periods of acute illness? including food modification appeared helpful in supporting nutritional status in several studies. No interventions aimed to improve oral hygiene.

Only one study included people with acute illness. It provided ONS during acute illness and reported no change in nutritional status [52].

What are the most effective ways to encourage people with dementia to eat, drink and maintain nutritional intake? Information provided here is supplemental to the main findings of this review, and overall evidence is weak or lacking - the review does not definitively show that any intervention is either useful or not useful

included studies. Due to lack of pre-registered protocols and details, most studies were judged at unclear risk of reporting and/or selection bias. Comparability of participants at baseline, suitability of outcomes measured and dementia diagnosis appeared to be satisfactory in most studies.

\section{Effects of interventions for swallowing problems Supplementary fruit juice}

These studies assessed the effects of providing additional energy and protein using ONS. Of 17 RCTs included, 11 assessed the effects of ONS plus usual food vs usual food alone on weight or body mass index (BMI), and reported

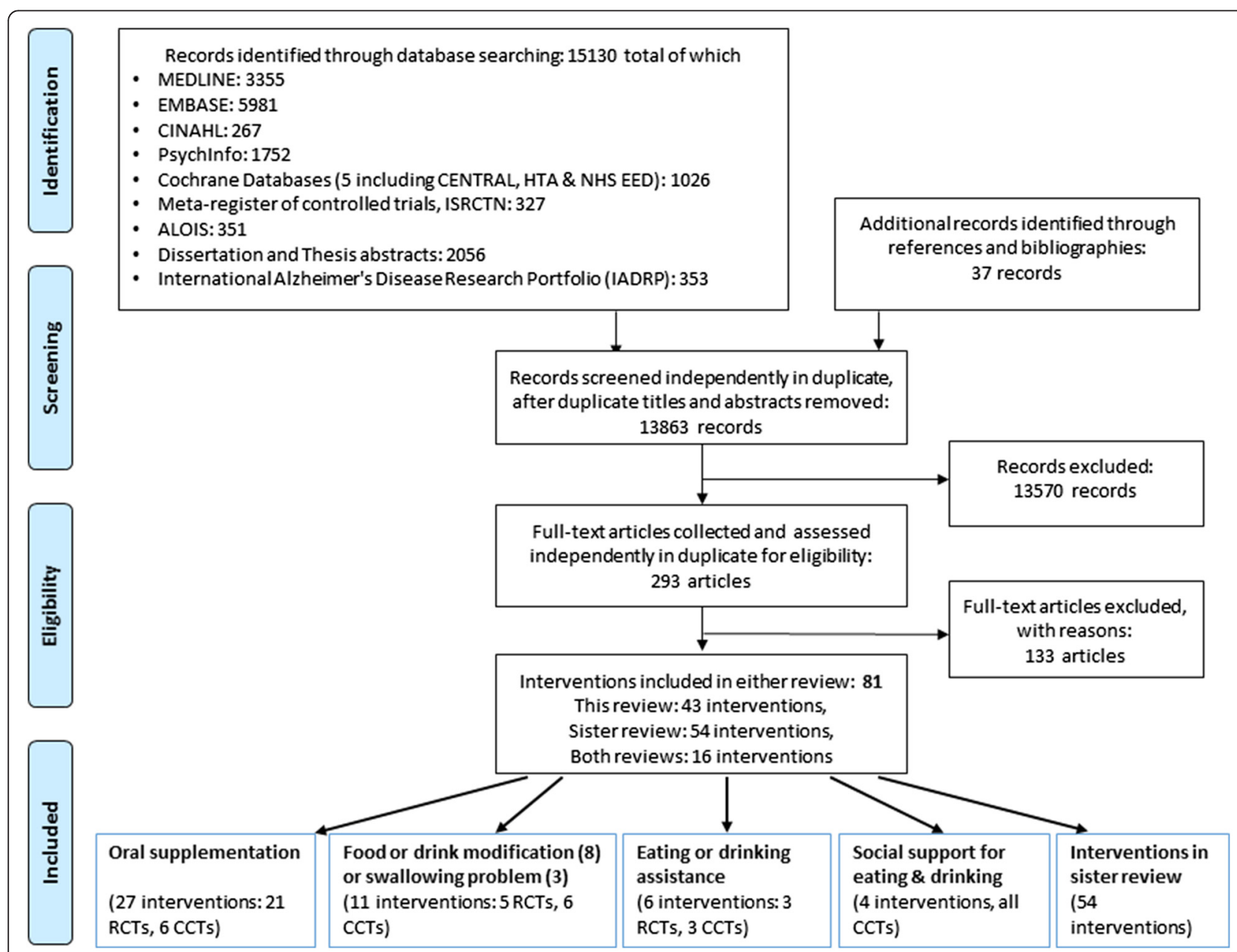

Fig. 1 EDWINA systematic review PRISMA flow diagram for studies of direct interventions. ${ }^{*}$ The number of interventions by category adds up to more than 43 (the total number of interventions in this paper) as several interventions were multicomponent, and so represented in several categories 
Table 2 Characteristics and results of included oral nutrition supplementation (ONS) interventions

\begin{tabular}{|c|c|c|c|c|c|c|c|c|c|}
\hline Study & Design & Setting, supplement type & $\begin{array}{l}\text { Number } \\
\text { completed }\end{array}$ & $\begin{array}{l}\text { Dementia } \\
\text { stage }\end{array}$ & $\begin{array}{l}\text { Dementia } \\
\text { type }\end{array}$ & $\begin{array}{l}\text { Effect on nutrition } \\
\text { or hydration status }\end{array}$ & $\begin{array}{l}\text { Effect on intake of } \\
\text { nutrients or fluid }\end{array}$ & $\begin{array}{l}\text { Quality and Other } \\
\text { outcomes }\end{array}$ & Duration \\
\hline \multicolumn{10}{|c|}{ ONS (including energy, protein and often other nutrients) plus usual food vs. usual food (with or without placebo ONS) } \\
\hline Abalan 1992 France [30] & RCT & $\begin{array}{l}\text { Geriatric inpatients. Proprietary ONS } \\
\text { ('Tonexis') vs usual food }\end{array}$ & $\begin{array}{l}I=15 \\
C=14\end{array}$ & NR & $A D$ & N/A & $\rightarrow$ E intake & $\uparrow$ Cognitive function & 15 weeks \\
\hline Beck 2002 Denmark [32] & RCT & $\begin{array}{l}\text { Nursing home (risk of malnourishment). } \\
\text { Home-made ONS vs usual food }\end{array}$ & $\begin{array}{l}I=8 \\
C=8\end{array}$ & NR & NR & $\rightarrow$ Weight & $\rightarrow$ E intake & N/A & 2 months \\
\hline Carlsson 2009 Sweden [34] & $\begin{array}{l}\mathrm{CCT} \\
(\mathrm{BA})\end{array}$ & $\begin{array}{l}\text { Group-living facilities for people with } \\
\text { dementia. Drinkable yogurt }\end{array}$ & 13 & NR & Mixed & $\downarrow$ Weight & $\begin{array}{l}\rightarrow \text { E intake } \\
\rightarrow \text { Fluid intake }\end{array}$ & $\rightarrow$ Functional status & 6 months \\
\hline Carver 1995 UK [36] & RCT & $\begin{array}{l}\text { Psychiatric hospital/elderly ward } \\
\text { (under-weight).Proprietary ONS ('Fortisip') } \\
\text { vs placebo }\end{array}$ & $\begin{array}{l}I=20 \\
C=20\end{array}$ & NR & NR & $\begin{array}{l}\uparrow \text { Weight } \\
\uparrow^{*} \text { BMl } \\
\rightarrow \text { TSF } \\
\uparrow \text { MAMC }\end{array}$ & $\mathrm{N} / \mathrm{A}$ & N/A & 12 weeks \\
\hline de Sousa 2012 Portugal [37] & RCT & $\begin{array}{l}\text { Psychiatric hospital, geriatric unit, mild } \\
\text { dementia patients (malnourished). } \\
\text { ONS vs usual care \& advice }\end{array}$ & $\begin{array}{l}I=20 \\
C=15\end{array}$ & Mild & $A D$ & $\begin{array}{l}\uparrow \text { Weight } \\
\uparrow \mathrm{BMl}\end{array}$ & $\downarrow$ Nutritional risk & $\begin{array}{l}\rightarrow \text { Functional status } \\
\rightarrow \text { Cognitive function }\end{array}$ & 3 weeks \\
\hline Faxen-Irving 2002 Sweden [38] & CCT & $\begin{array}{l}\text { Group-living for people with dementia. } \\
\text { ONS \& diet advice vs usual care }\end{array}$ & $\begin{array}{l}I=21 \\
C=12\end{array}$ & Mixed & Mixed & $\begin{array}{l}\uparrow^{a} \text { Weight } \\
\uparrow^{a} \mathrm{BMI} \\
\uparrow \neq \mathrm{TSF} \\
\rightarrow \mathrm{AMC}\end{array}$ & $\mathrm{N} / \mathrm{A}$ & 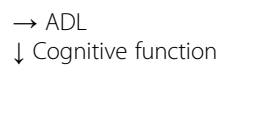 & 5 months \\
\hline $\begin{array}{l}\text { FICSIT Fiatarone Singh } 2000 \text { USA [39, } \\
86-88]\end{array}$ & RCT & $\begin{array}{l}\text { Nursing home (long term rehabilitation } \\
\text { centre). } \\
\text { ONS vs placebo }\end{array}$ & $\begin{array}{l}I=24 \\
C=26\end{array}$ & NR & NR & $\begin{array}{l}\uparrow \text { Weight } \\
\uparrow \mathrm{BMl} \\
\rightarrow \mathrm{MAMA} \\
\rightarrow \mathrm{TBW}\end{array}$ & $\begin{array}{l}\rightarrow \text { E intake } \\
\rightarrow \text { Fluid intake }\end{array}$ & $\rightarrow$ Functional status & 10 weeks \\
\hline $\begin{array}{l}\text { FOPANU Carlsson } 2011 \text { Sweden } \\
{[35,89,90]}\end{array}$ & RCT & $\begin{array}{l}\text { Residential care facilities.Protein-enriched } \\
\text { drink (+/- exercise) vs placebo drink } \\
(+/- \text { exercise) }\end{array}$ & $\begin{array}{l}I=96 \\
C=95\end{array}$ & NR & NR & $\begin{array}{l}\rightarrow \text { Weight } \\
\rightarrow \mathrm{ICW}\end{array}$ & N/A & $\begin{array}{l}\rightarrow \text { Balance } \\
\rightarrow \text { Gait } \\
\rightarrow \text { Lower limb strength }\end{array}$ & 3 months \\
\hline Gregorio 2003 Spain [40] & $\mathrm{RCT}$ & $\begin{array}{l}\text { Nursing home residents with AD. } \\
\text { Proprietary ONS ('Nutrison') vs usual food }\end{array}$ & $\begin{array}{l}\mathrm{I}=24 \\
\mathrm{C}=74\end{array}$ & Mod & $A D$ & $\uparrow^{*} \mathrm{BMl}$ & $\begin{array}{l}\downarrow^{*} \text { Nutritional } \\
\text { risk }\end{array}$ & $\mathrm{N} / \mathrm{A}$ & 12 months \\
\hline Lauque 2000 France [44] & RCT & $\begin{array}{l}\text { Nursing homes (risk of malnourishment). } \\
\text { Proprietary ONS (Clinutren, Nestle) } \\
\text { vs usual food }\end{array}$ & $\begin{array}{l}I=19 \\
C=22\end{array}$ & NR & NR & $\begin{array}{l}\rightarrow \text { Weight } \\
\rightarrow \text { BMl }\end{array}$ & $\begin{array}{l}\uparrow \text { E intake } \\
\uparrow \text { Protein intake }\end{array}$ & $\rightarrow$ Grip strength & 2 months \\
\hline Lauque 2004 France [45] & $\mathrm{RCT}$ & $\begin{array}{l}\text { Geriatric wards \& day centres } \\
\text { (risk of malnourishment). Proprietary } \\
\text { ONS (Clinutren) vs usual food }\end{array}$ & $\begin{array}{l}I=37 \\
C=43\end{array}$ & Mod & NR & $\uparrow$ Weight $\uparrow \mathrm{BMI}$ & $\begin{array}{l}\uparrow \text { E intake } \\
\downarrow \text { Nutritional risk }\end{array}$ & $\begin{array}{l}\rightarrow \mathrm{ADL} \\
\rightarrow \text { Cognitive function } \\
\rightarrow \text { Eating behaviour }\end{array}$ & 3 months \\
\hline Manders 2009 Netherlands [46] & RCT & Nursing homes.ONS vs placebo & $\begin{array}{l}I=78 \\
C=33\end{array}$ & NR & NR & $\begin{array}{l}\uparrow \text { Weight } \\
\uparrow \text { Calf circumference }\end{array}$ & N/A & $\begin{array}{l}\rightarrow \text { Functional status } \\
\rightarrow \text { Grip strength } \\
\rightarrow \text { Cognitive status }\end{array}$ & 24 weeks \\
\hline Navrátilová 2007 Czech Republic [47] & RCT & $\begin{array}{l}\text { Institutionalised residents with AD } \\
\text { (type of institution unclear).Proprietary ONS } \\
\text { ('Nutridrink') vs usual food }\end{array}$ & $\begin{array}{l}I=50 \\
C=50\end{array}$ & NR & $A D$ & $\begin{array}{l}\rightarrow \text { Weight } \\
\rightarrow \text { BMl }\end{array}$ & $\begin{array}{l}\uparrow^{\dagger} E \text { intake } \\
\uparrow^{\dagger} \text { Protein intake }\end{array}$ & $\uparrow$ Cognitive function & 1 year \\
\hline Pivi 2011 Brazil [55] & $\mathrm{RCT}$ & $\begin{array}{l}\text { Setting unclear. Proprietary supplement } \\
\text { (Ensure with FOS }{ }^{\bullet} \text { ) vs usual care }\end{array}$ & $\begin{array}{l}I=26 \\
C=27\end{array}$ & $\begin{array}{l}\text { Mild- } \\
\text { severe }\end{array}$ & $A D$ & $\begin{array}{l}\uparrow^{*} \text { Weight } \\
\uparrow^{*} \mathrm{BMl} \\
\uparrow^{*} \mathrm{AMC} \\
\uparrow^{*} \mathrm{AC} \rightarrow \mathrm{TSF}\end{array}$ & N/A & N/A & 6 months \\
\hline Planas 2004 Spain [48] & $\begin{array}{l}C C T \\
(B A)\end{array}$ & $\begin{array}{l}\text { Dementia care day centre.ONS +/- } \\
\text { micronutrients }\end{array}$ & $\begin{array}{l}I=23 \\
C=21\end{array}$ & Mild & $A D$ & $\begin{array}{l}\rightarrow \mathrm{BMI} \\
\uparrow \mathrm{MAMC} \\
\uparrow \mathrm{TSF}\end{array}$ & $\uparrow \mathrm{E}$ intake & $\rightarrow$ Cognitive function & 6 months \\
\hline
\end{tabular}


Table 2 Characteristics and results of included oral nutrition supplementation (ONS) interventions (Continued)

\begin{tabular}{|c|c|c|c|c|c|c|c|c|c|}
\hline Study & Design & Setting, supplement type & $\begin{array}{l}\text { Number } \\
\text { completed }\end{array}$ & $\begin{array}{l}\text { Dementia } \\
\text { stage }\end{array}$ & $\begin{array}{l}\text { Dementia } \\
\text { type }\end{array}$ & $\begin{array}{l}\text { Effect on nutrition } \\
\text { or hydration status }\end{array}$ & $\begin{array}{l}\text { Effect on intake of } \\
\text { nutrients or fluid }\end{array}$ & $\begin{array}{l}\text { Quality and Other } \\
\text { outcomes }\end{array}$ & Duration \\
\hline Simmons 2010a USA [50] & $\mathrm{RCT}$ & $\begin{array}{l}\text { Long-term care facilities, type unclear. } \\
\text { Between meal nutritional supplements vs. } \\
\text { usual care }\end{array}$ & $\begin{array}{l}I=18 \\
C=20\end{array}$ & NR & NR & $\rightarrow$ Weight & $\rightarrow$ E intake & $\uparrow$ Costs \& staff time & 6 weeks \\
\hline $\begin{array}{l}\text { Souvenir I Scheltens } 2010 \text { USA \& } \\
\text { Europe }[49,91-96]\end{array}$ & $\begin{array}{l}C C T \\
(B A)+\infty\end{array}$ & $\begin{array}{l}\text { AD Treatment Centres.Proprietary ONS } \\
\text { (Souvenaid) vs isocaloric placebo }\end{array}$ & $\begin{array}{l}I=98 \\
C=97\end{array}$ & Mild & AD & $\rightarrow \mathrm{BMl}$ & N/A & $\begin{array}{l}\rightarrow \text { Cognitive function } \\
\rightarrow \text { QoL }\end{array}$ & $\begin{array}{l}12 \& \\
24 \text { weeks }\end{array}$ \\
\hline $\begin{array}{l}\text { Stange } 2013 \text { Germany } \\
{[51,97-99]}\end{array}$ & RCT & $\begin{array}{l}\text { Nursing home (risk of malnutrition).ONS vs } \\
\text { usual care }\end{array}$ & $\begin{array}{l}I=45 \\
C=42\end{array}$ & $\begin{array}{l}\text { Mod- } \\
\text { severe }\end{array}$ & NR & $\begin{array}{l}\uparrow \text { Weight } \\
\rightarrow \mathrm{BMI} \\
\uparrow \cup \mathrm{UC} \\
\uparrow \text { Calf circumference }\end{array}$ & $\begin{array}{l}\rightarrow \text { E intake } \\
\rightarrow \text { Protein intake } \\
\rightarrow \text { Nutritional risk }\end{array}$ & $\begin{array}{l}\rightarrow \text { Cognitive function } \\
\rightarrow \mathrm{ADL}\end{array}$ & 12 weeks \\
\hline $\begin{array}{l}\text { Wouters-Wesseling } 2002 \text { Netherlands } \\
\text { [53] }\end{array}$ & $\mathrm{RCT}$ & $\begin{array}{l}\text { Nursing homes, residents with dementia.ONS } \\
\text { vs placebo }\end{array}$ & $\begin{array}{l}I=19 \\
C=16\end{array}$ & NR & Mixed & $\begin{array}{l}\uparrow \text { Weight } \\
\rightarrow \mathrm{BMl}\end{array}$ & N/A & $\rightarrow$ Functional status & 12 weeks \\
\hline $\begin{array}{l}\text { Wouters-Wesseling } 2006 \text { Netherlands } \\
\text { [52] }\end{array}$ & RCT & $\begin{array}{l}\text { Psychogeriatric nursing homes (with acute } \\
\text { infection).ONS vs usual care }\end{array}$ & $\begin{array}{l}I=18 \\
C=16\end{array}$ & NR & NR & $\begin{array}{l}\rightarrow \text { Weight } \\
\rightarrow \text { TST } \\
\rightarrow \text { AMC }\end{array}$ & $\rightarrow$ E intake & $\rightarrow$ Functional status & 5 weeks \\
\hline $\begin{array}{l}\text { Young } 2004 \text { Canada } \\
{[54,62,100]}\end{array}$ & $\mathrm{RCT}$ & $\begin{array}{l}\text { Dementia units within a nursing home. ONS } \\
\text { vs high carbohydrate meals }\end{array}$ & $\begin{array}{l}I=15 \\
C=19\end{array}$ & NR & AD & $\uparrow$ Weight & $\begin{array}{l}\uparrow \text { E intake } \\
\uparrow \text { Protein intake }\end{array}$ & N/A & 3 weeks \\
\hline \multicolumn{10}{|c|}{ Fruit juice plus normal food vs control drink plus normal food } \\
\hline Krikorian 2010a USA [42] & $\mathrm{RCT}$ & Community-dwelling.Grape juice vs placebo & $\begin{array}{l}I=5 \\
C=7\end{array}$ & $\mathrm{MCl}$ & N/A & $\begin{array}{l}\rightarrow^{*} \text { Weight } \\
\rightarrow^{*} \text { Waist }\end{array}$ & N/A & $\begin{array}{l}\uparrow \text { Learning, } \\
\rightarrow \text { Spatial awareness, } \\
\rightarrow \text { Recall }\end{array}$ & 12 weeks \\
\hline Krikorian 2010b USA [43] & ССТ & $\begin{array}{l}\text { Community-dwelling.Blueberry juice vs } \\
\text { placebo }\end{array}$ & $\begin{array}{l}I=9 \\
C=7\end{array}$ & $\mathrm{MCl}$ & N/A & $\begin{array}{l}\rightarrow^{*} \text { Weight } \\
\rightarrow^{*} \text { Waist }\end{array}$ & N/A & $\begin{array}{l}\text { ? Cognition, } \\
\text { ? Spatial awareness }\end{array}$ & 12 weeks \\
\hline Krikorian 2012 USA [41] & $\mathrm{RCT}$ & Community-dwelling.Grape juice vs placebo & $\begin{array}{l}I=10 \\
C=11\end{array}$ & $\mathrm{MCl}$ & N/A & $\begin{array}{l}\rightarrow \text { Weight } \\
\rightarrow \text { Waist }\end{array}$ & N/A & NR & 16 weeks \\
\hline \multicolumn{10}{|c|}{ Additional snacks between meals plus usual food vs usual food } \\
\hline Simmons 2010b USA [50] & $\mathrm{RCT}$ & $\begin{array}{l}\text { Long-term care facilities.Between meal snacks } \\
\& \text { assistance vs usual care }\end{array}$ & $\begin{array}{l}I=25 \\
C=20\end{array}$ & NR & NR & $\rightarrow$ Weight & $\rightarrow$ E intake & $\begin{array}{l}\uparrow \text { Costs } \\
\uparrow \text { Staff time }\end{array}$ & 6 weeks \\
\hline \multicolumn{10}{|c|}{ Multicomponent interventions including ONS } \\
\hline Beck 2010 Denmark [31, 101] & RCT & $\begin{array}{l}\text { Elderly nursing home residents.ONS, Gratin } \\
\text { diet, exercise, oral care vs usual care }\end{array}$ & $\begin{array}{l}I=54 \\
C=55\end{array}$ & NR & NR & $\begin{array}{l}\uparrow \text { Weight } \\
\uparrow \mathrm{BMl}\end{array}$ & $\begin{array}{l}\rightarrow \text { E intake } \\
\uparrow \text { Protein intake }\end{array}$ & $\begin{array}{l}\rightarrow \text { Cognitive performance } \\
\rightarrow \mathrm{ADL}\end{array}$ & 11 weeks \\
\hline Boffelli 2004 Italy [33] & $\begin{array}{l}\mathrm{CCT} \\
(\mathrm{BA})\end{array}$ & $\begin{array}{l}\text { Malnourished residents of dementia unit. } \\
\text { Individualised diet including mealtime } \\
\text { assistance, environmental modification and } \\
\text { ONS if required }\end{array}$ & 19 & Severe & Mixed & $\begin{array}{l}\rightarrow \text { Weight } \\
\rightarrow \text { BMl } \\
\uparrow \text { Serum albumin }\end{array}$ & N/A & N/A & 18 months \\
\hline
\end{tabular}

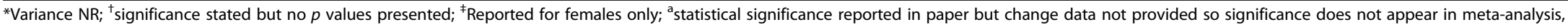
- these were RCTs, but we used their data as before-after comparisons, so they are reported here as BA. $\uparrow$ indicates statistically significant increase; $\downarrow$ indicates statistically significant reduction; $\rightarrow$ indicates no statistically significant effect; statistical significance of all effects were checked by reviewers where data were

$A D$ Alzheimer's disease, $A C$ Arm Circumference, $A D L$ activities of daily living, $A M C$ arm muscle circumference, $B A$ before-after or pre-post, $B M I$ body mass index, $C$ control group, CCT controlled clinical trial (with a

concurrent control arm unless indicated as BA), CDR Clinical Dementia Rating Scale, C control group, E energy, GDS Global Deterioration Scale, I intervention group, ICW intracellular water, MAMA mid-arm muscle area, $M A M C$ mid-arm muscle circumference, $M C l$ mild cognitive impairment, mod moderate, N/A not applicable, NR not reported, ONS oral nutritional supplement, QoL quality of life, $R C T$ randomised controlled trial, suppl supplement, TSF triceps skin fold, TST triceps skin fold thickness, TBW total body water, UAC upper arm circumference, vs versus 
Table 3 Characteristics and results of included food and drink modification, swallowing intervention, eating or drinking assistance and social support interventions

\begin{tabular}{|c|c|c|c|c|c|c|c|c|c|}
\hline Study & Design & Setting, Intervention type & No. & $\begin{array}{l}\text { Dementia } \\
\text { stage }\end{array}$ & $\begin{array}{l}\text { Dementia } \\
\text { type }\end{array}$ & $\begin{array}{l}\text { Effect on } \\
\text { Nutrition / } \\
\text { hydration status }\end{array}$ & Intake effect & Quality \& other outcomes & Duration \\
\hline \multicolumn{10}{|l|}{ Swallowing interventions } \\
\hline $\begin{array}{l}\text { Bautmans } 2008 \\
\text { Belgium [56] }\end{array}$ & $\mathrm{RCT}$ & $\begin{array}{l}\text { Nursing home. Cervical spine } \\
\text { mobilization to help dysphagia }\end{array}$ & 15 & Severe & $A D$ & NR & NR & $\uparrow$ Dysphagia limit & 1 week \\
\hline $\begin{array}{l}\text { Germain } 2006 \\
\text { Canada [57] }\end{array}$ & $\mathrm{RCT}$ & $\begin{array}{l}\text { Long term care facility. Dysphagia } \\
\text { diet }\end{array}$ & $\begin{array}{l}I=8 \\
C=9\end{array}$ & NR & $\begin{array}{l}A D \\
\text { \&others }\end{array}$ & $\uparrow$ Weight & $\uparrow E$ intake & NR & 12 weeks \\
\hline $\begin{array}{l}\text { Robbins } 2008 \text { USA } \\
{[58,102-104]}\end{array}$ & $\mathrm{RCT}$ & $\begin{array}{l}\text { Hospitals \& nursing homes. } \\
\text { 1. Nectar-thick or } \\
\text { 2. Honey-thick consistency fluids } \\
\text { 3. Chin-tuck position }\end{array}$ & $\begin{array}{l}\text { Nectar 133, } \\
\text { Honey 123, } \\
\text { Chin-tuck } \\
259\end{array}$ & Various & NR & & NR & $\begin{array}{l}\rightarrow \text { Aspiration pneumonia incidence (for } \\
\text { thickened vs chin-tuck) }\end{array}$ & 3 months \\
\hline \multicolumn{10}{|l|}{ Food modification } \\
\hline $\begin{array}{l}\text { Beck } 2010 \text { Denmark } \\
{[31,101]}\end{array}$ & $\mathrm{RCT}$ & $\begin{array}{l}\text { Elderly nursing home residents. ONS, } \\
\text { Gratin diet, swallowing problem } \\
\text { management, exercise and oral care } \\
\text { vs usual care }\end{array}$ & $\begin{array}{l}I=54 \\
C=55\end{array}$ & NR & NR & $\begin{array}{l}\uparrow \text { BMl } \\
\uparrow \text { Weight }\end{array}$ & $\begin{array}{l}\overrightarrow{\text { E-intake }} \\
\uparrow \text { Protein intake }\end{array}$ & $\begin{array}{l}\rightarrow \text { Cognitive performance } \\
\rightarrow \mathrm{ADL}\end{array}$ & 11 weeks \\
\hline $\begin{array}{l}\text { Boffelli } 2004 \text { Italy } \\
\text { [33] }\end{array}$ & $\mathrm{CCT}(\mathrm{BA})$ & $\begin{array}{l}\text { Dementia unit. Diet \& environment } \\
\text { modification, feeding assistance and } \\
\text { supplements }\end{array}$ & 29 & Severe & Various & $\begin{array}{l}\rightarrow \text { BMl } \\
\rightarrow \text { weight } \\
\uparrow \text { Albumin }\end{array}$ & NR & NR & 18 months \\
\hline Jean 1997 USA [59] & $C C T(B A)$ & Nursing home.Finger food menu & 12 & NR & $\begin{array}{l}A D \\
\text { \&others }\end{array}$ & ? Weight loss arrest & NR & ? Eating independence & 6 months \\
\hline $\begin{array}{l}\text { Keller } 2003 \text { Canada } \\
{[63,105]}\end{array}$ & $\mathrm{CCT}$ & $\begin{array}{l}\text { Long term care facilities. } \\
\text { Individualised food service, food } \\
\text { modification, education and dietitian } \\
\text { time }\end{array}$ & $\begin{array}{l}I=33 \\
C=49\end{array}$ & NR & $\begin{array}{l}A D \\
\text { \&others }\end{array}$ & $\uparrow$ weight & NR & NR & 21 months \\
\hline $\begin{array}{l}\text { Kenkmann } 2010 \text { UK } \\
{[64,106]}\end{array}$ & $\mathrm{RCT}$ & $\begin{array}{l}6 \text { Care homes. Dining environment } \\
\& \text { menu changes }\end{array}$ & $\begin{array}{l}I=57 \\
C=48\end{array}$ & NR & NR & $\begin{array}{l}\rightarrow \text { Weight, } \\
\rightarrow \text { BMl, } \\
\rightarrow \text { Hydrated }\end{array}$ & NR & $\rightarrow$ Enjoymentof food/drink & 1 year \\
\hline $\begin{array}{l}\text { Salas-Salvado } 2005 \\
\text { Spain [61] }\end{array}$ & $\mathrm{RCT}$ & $\begin{array}{l}\text { Geriatric institutions. Meal } \\
\text { replacement with commercial } \\
\text { lyophilised supplement }\end{array}$ & $\begin{array}{l}I=15 \\
C=23\end{array}$ & Severe & $A D$ & $\begin{array}{l}\uparrow \text { Weight } \\
\uparrow \text { Serum albumin }\end{array}$ & $\begin{array}{l}\rightarrow \text { E intake } \\
\rightarrow \text { Nutritional risk }\end{array}$ & $\begin{array}{l}\rightarrow \text { Eating behaviour } \\
\rightarrow \text { Mortality } \\
\rightarrow \text { Cognitive parameters }\end{array}$ & 3 months \\
\hline $\begin{array}{l}\text { Soltesz } 1995 \text { USA } \\
\text { [60] }\end{array}$ & $\mathrm{CCT}(\mathrm{BA})$ & $\begin{array}{l}\text { Alzheimer's Care Centre. } \\
\text { Finger food provision }\end{array}$ & 43 & NR & $A D$ & $\rightarrow$ Weight & $\begin{array}{l}\uparrow \text { Proportion } \\
\text { food eaten }\end{array}$ & NR & 6 months \\
\hline $\begin{array}{l}\text { Young } 2005 \text { Canada } \\
{[54,62,100]}\end{array}$ & $\mathrm{RCT}$ & $\begin{array}{l}\text { Nursing home. } \\
\text { High CHO dinners }\end{array}$ & $\begin{array}{l}I=15 \\
C 19\end{array}$ & NR & $A D$ & NR & $\uparrow E$ intake & NR & 21 days \\
\hline
\end{tabular}


Table 3 Characteristics and results of included food and drink modification, swallowing intervention, eating or drinking assistance and social support interventions (Continued)

\begin{tabular}{|c|c|c|c|c|c|c|c|c|c|}
\hline Study & Design & Setting, Intervention type & No. & $\begin{array}{l}\text { Dementia } \\
\text { stage }\end{array}$ & $\begin{array}{l}\text { Dementia } \\
\text { type }\end{array}$ & $\begin{array}{l}\text { Effect on } \\
\text { Nutrition / } \\
\text { hydration status }\end{array}$ & Intake effect & Quality \& other outcomes & Duration \\
\hline \multicolumn{10}{|c|}{ Eating or drinking assistance interventions } \\
\hline $\begin{array}{l}\text { Boffelli } 2004 \text { Italy } \\
\text { [33] }\end{array}$ & $\mathrm{CCT}(\mathrm{BA})$ & $\begin{array}{l}\text { Dementia unit, diet \& environment } \\
\text { modification, feeding assistance and } \\
\text { supplements }\end{array}$ & 29 & Severe & Various & $\begin{array}{l}\rightarrow \text { BMl } \\
\rightarrow \text { Weight } \\
\uparrow \text { Albumin }\end{array}$ & NR & NR & 18 months \\
\hline $\begin{array}{l}\text { Simmons } 2001 \text { USA } \\
{[65,107]}\end{array}$ & $C \subset \mathrm{T}$ & $\begin{array}{l}\text { Nursing Homes. Staff assistance, } \\
\text { prompting, food/drink service and } \\
\text { exercise }\end{array}$ & $\begin{array}{l}I=48 \\
C=15\end{array}$ & $N R$ & NR & $\begin{array}{l}\rightarrow \text { Serum osmolality } \\
\rightarrow \text { BUN: creatinine ratio }\end{array}$ & $\begin{array}{l}\rightarrow \text { Food\& fluid } \\
\text { intake }\end{array}$ & NR & 32 weeks \\
\hline $\begin{array}{l}\text { Simmons } 2008 \text { USA } \\
\text { [66] }\end{array}$ & RCT & $\begin{array}{l}\text { Skilled nursing homes. Either meal } \\
\text { time or between meal feeding } \\
\text { assistance }\end{array}$ & $\begin{array}{l}I=35 \\
C=34\end{array}$ & $N R$ & $N R$ & $\begin{array}{l}\text { ? BMI, } \\
\text { ? Weight }\end{array}$ & $\uparrow E$ intake & NR & 24 weeks \\
\hline $\begin{array}{l}\text { Simmons 2010a } \\
\text { USA [50] }\end{array}$ & RCT & $\begin{array}{l}\text { Long-term care facilities. Between } \\
\text { meal supplements \& assistance vs } \\
\text { usual care }\end{array}$ & $\begin{array}{l}11=18 \\
C 20\end{array}$ & $N R$ & $N R$ & $\rightarrow$ Weight & $\rightarrow$ E intake & NR & 6 weeks \\
\hline $\begin{array}{l}\text { Simmons 2010b } \\
\text { USA [50] }\end{array}$ & RCT & $\begin{array}{l}\text { Long-term care facilities. Between } \\
\text { meal snacks \& assistance vs usual } \\
\text { care }\end{array}$ & $\begin{array}{l}12=25 \\
C=20\end{array}$ & NR & $N R$ & $\rightarrow$ Weight & $\rightarrow$ E intake & NR & 6 weeks \\
\hline $\begin{array}{l}\text { Wong } 2008 \mathrm{New} \\
\text { Zealand [67] }\end{array}$ & $\mathrm{CCT}(\mathrm{BA})$ & $\begin{array}{l}\text { Short stay assessment unit.Individual } \\
\text { mealtime assistance }\end{array}$ & 7 & NR & NR & $\uparrow \mathrm{BMl}$ & $\uparrow E$ intake & NR & 12 weeks \\
\hline \multicolumn{10}{|c|}{ Studies with a strong social element around eating/drinking } \\
\hline Altus 2002 USA [68] & $C C T(B A)$ & $\begin{array}{l}\text { Locked dementia unit. } \\
\text { Family-style meals }-/+ \text { staff training }\end{array}$ & 5 & $\begin{array}{l}\text { Mod- } \\
\text { severe }\end{array}$ & $\begin{array}{l}\mathrm{AD} \\
\text { \&others }\end{array}$ & $N R$ & NR & $\begin{array}{l}\text { ? Resident Participation in mealtime tasks } \\
\text { ? Appropriate communication } \\
\text { ? Praise statements } \\
\text { ? Staff satisfaction with resident participation }\end{array}$ & $\begin{array}{l}5 \text { days each } \\
\text { period }\end{array}$ \\
\hline $\begin{array}{l}\text { Charras } 2010 \text { France } \\
\text { [69] }\end{array}$ & CCT & $\begin{array}{l}\text { Dementia units in nursing homes. } \\
\text { Shared mealtime with staff }\end{array}$ & $\begin{array}{l}I=8 \\
C=10\end{array}$ & Severe & $A D$ & $\uparrow$ Weight & NR & $\begin{array}{l}\text { ? Autonomy } \\
\text { ? Quality of interactions } \\
\text { ? Food quality }\end{array}$ & 6 months \\
\hline $\begin{array}{l}\text { Huang } 2009 \text { Taiwan } \\
\text { [70] }\end{array}$ & $\mathrm{CCT}(\mathrm{BA})$ & $\begin{array}{l}\text { Older person care facility, } \\
\text { Reminiscence cooking therapy }\end{array}$ & 12 & Mild-mod & NR & NR & NR & $\begin{array}{l}\rightarrow \text { MMSE } \\
\uparrow \text { Happiness } \\
\rightarrow \text { Communication } \\
\text { ? Participation }\end{array}$ & 8 weeks \\
\hline $\begin{array}{l}\text { Santo Pietro } 1998 \\
\text { USA [71] }\end{array}$ & CCT & $\begin{array}{l}\text { Dementia unit within a nursing } \\
\text { home. } \\
\text { Breakfast club (communication } \\
\text { therapy) }\end{array}$ & $\begin{array}{l}I=20 \\
C=20\end{array}$ & Mild-mod & $A D$ & $N R$ & NR & $\begin{array}{l}\uparrow \text { Interest \& involvement } \\
\uparrow \text { Communication }\end{array}$ & 12 weeks \\
\hline
\end{tabular}

$\uparrow$ indicates statistically significant increase; $\downarrow$ indicates statistically significant reduction; $\rightarrow$ indicates no statistically significant effect; ? indicates unclear whether effect was statistically significant. Statistical significance of all effects were checked by reviewers where data were available, and reviewers results used when they differed from the original paper.

$A D$ Alzheimer's disease, $A D L$ activities of daily living, BA before-after, BMI body mass index, CCT controlled clinical trial, CG control group, CHO Carbohydrate, E energy, IG intervention group, NR not reported, ONS oral nutritional supplement, $R C T$ randomised controlled trial, vs versus 
results that could be incorporated into meta-analysis. Duration of three was $>12$ weeks and meta-analysis suggested no statistically significant effect on weight (mean difference [MD] $0.72 \mathrm{~kg}, 95 \%$ CI -1.02-2.45, 382 participants) but with high heterogeneity ( $\mathrm{I}^{2} 89 \%$ ) (Fig. 2). One study [45] also assessed effects on BMI, suggesting a small, statistically significant, increase of $0.64 \mathrm{~kg} / \mathrm{m}^{2}$ (95\% CI 0.22-1.06, 80 participants) (Fig. 3).

Eight RCTs assessed effects over 3-12 weeks. Metaanalysis suggested small, statistically significant, effects of supplementation on weight (2.02 kg, $95 \%$ CI 1.53-2.50, 344 participants, $\mathrm{I}^{2} 0 \%$, Fig. 2) and BMI $\left(0.91 \mathrm{~kg} / \mathrm{m}^{2}, 95 \%\right.$ CI 0.56-1.25, $\mathrm{I}^{2} 0$ \%, 221 participants, Fig. 3). Six RCTs either did not report weight or BMI [30], suggested no effect [32, $40,47]$, or reported improvements but presented data in a form that could not be included in meta-analysis $[48,54]$. We did not include CCT results in meta-analyses as they provide less valid data on effects. One CCT [38] reported significant weight gain $(3.5 \mathrm{~kg})$ compared to control after 5 months ONS ( $\mathrm{p}=0.003$ ) while two RCTs $[48,49]$ (analysed as before-after [BA] assessing effects of ONS compared to baseline) suggested no effect at six months on BMI.

Effects on other anthropometric measures were mixed: One RCT [36] reported positive effect on Mid-upper Arm Muscle Circumference but not on Triceps Skinfold Thickness; in three RCTs $[46,51,55]$ Arm Muscle Circumference and/or calf circumference improved significantly; in a small Dutch RCT [52] there was no effect on Triceps Skinfold Thickness or Arm Muscle Circumference. One CCT [48] reported significantly increased Triceps Skinfold Thickness and Mid-Upper Arm Circumference.

Two RCTs [37, 45] assessed effects on Mini-Nutrition Assessment (MNA), and meta-analysis suggested significant improvement with supplementation (1.41 points, $95 \%$ CI $1.06-1.75, \mathrm{I}^{2} 0 \%, 115$ participants, see Additional file 3: Figure S3.2). Meta-analysis of six RCTs [39, 44, 45, 50-52] suggested small statistically significant increases in energy intake with ONS of $132 \mathrm{kcal} /$ day (95 \% CI 41-223, 279 participants, $\mathrm{I}^{2} 14 \%$, Additional file 3: Figure S3.3).

Quality of life was measured in one RCT [51] with no significant effect on any of nine sub-scales for quality of life, except 'positive self-perception' $(p=0.011)$, and no effect on quality of life was found in the single CCT [49]. Functional status was assessed in several RCTs and CCTs but none found any effect (Table 2). Cognitive status was assessed in five RCTs and three CCTs. Three RCTs and all the CCTs found no effect [37, 38, 45, 48, 49, 51], while one RCT [30] reported an improvement of 6 points in the MMSE score $(p<0.001$, a surprising finding) and another [47] reported slowing the rate of cognitive decline $(-1.3$ vs. $-3.7, p=0.024)$. Hydration status was measured in one RCT [39] which reported no effect.

Five RCTs [35, 37, 39, 40,53] reported 40 deaths in 419 participants, but meta-analysis did not suggest any effect of supplementation on mortality (RR 0.83, $95 \%$ CI 0.43-1.61, Additional file 3: Figure S3.4).

\section{Effects of food and drink modification Additional snacks between meals}

Three studies assessed effects of fruit juice plus normal food vs control drink plus normal food. Two small RCTs $[41,42]$ examined effects of daily grape juice vs placebo for 12 or 16 weeks in community dwelling older adults with MCI, finding no significant effect on weight or waist circumference. One CCT [43] found no effect of 12 weeks of blueberry juice vs placebo in nine adults with MCI on weight, but suggested improvement of some memory tests (no clear numerical data presented, and control data utilised from another study) [42].

\section{Effects of eating and drinking assistance Multicomponent interventions including ONS}

A US RCT investigating energy intake and cost effectiveness of between-meal nutritious snacks in 45 nursing home residents [50] found no significant effect at five weeks on weight $(p=0.66)$, or energy intake $(p=0.20)$. Staff time increased (by 1.7 minutes/resident/offer), as did product and staff costs $(p<0.01)$. A CCT [34] on 15 people living in Swedish dementia facilities reported significant weight loss after six months drinkable probiotic yogurt (-3.9 kg, SD 3.9, range -9.2-3), but no change in energy or fluid intake.

\section{Effects of interventions with a strong social element around eating and drinking}

One RCT [31] tested effects of 11 weeks of ONS, management of swallowing problems with soft pureed gratin diet, oral care and exercise vs usual care in 121 Danish dementia nursing home residents, finding improved weight ( $1.3 \%$ vs. $-0.6 \%, \mathrm{p}=0.005)$ and BMI $(0.4 \%$ vs. $-0.2 \%, p=$ $0.003)$. Energy intake (0.7 vs. $-0.3 \mathrm{MJ} /$ day, $p=0.084$ ), functional and cognitive status did not alter. A CCT [35] evaluated diet and environmental modification, increased nurse time and assistance at meals in 19 malnourished Italian dementia unit residents, finding increased serum albumin (3.0 to $3.4 \mathrm{~g} / \mathrm{dL}$ at 18 months) but no changes in weight or BMI.

\section{Effects of interventions for swallowing problems}

Swallowing interventions included interventions aiming to assess, treat or manage swallowing problems. Three RCTs [56-58] assessed effectiveness of interventions to support people with swallowing problems, two had low risk of selection bias $[57,58]$, all three had high or unclear risk of performance bias and one [56] was at low risk of detection bias (Additional file 3: Figure S3.5). None was at low risk of bias overall, and meta-analysis was not possible due to differences in interventions. 
One RCT [57] examined effectiveness of a dysphagia diet (reformed minced/pureéd foods and thickened fluids) vs standard diet in 17 cognitively impaired residents with weight loss and swallowing problems (their stage of dementia was not defined). Weight $(+3.9 \mathrm{~kg}$ vs. $-0.79 \mathrm{~kg}$, $p=0.02)$ and energy intake increased $(p=0.03)$. The second RCT [58] compared thickened liquids vs chindown drinking in aspirating patients (nutritional status and dementia stage were not defined) finding no differences in aspiration pneumonia incidence or death (with no control group, it is unclear whether interventions were effective overall).

The third RCT [56] assessed one week cervical-spine mobilization vs therapist socialising visits in 15 people with severe AD and dysphagia. Dysphagia limit (maximal bolus of water swallowed in a single movement) increased from 3 to $10 \mathrm{ml}$ (median), significantly greater than control $(p=0.03)$, but fluid intake and hydration status were not measured.

\section{Effects of food and drink modification}

Food and drink modification included any modification to the food/drink itself, including nutritional or textural alterations and finger foods (except specifically for those with swallowing problems). All eight interventions were at high or unclear risk of selection, performance and detection biases, so none were at low risk of bias overall (Additional file 3: Figure S3.6. Meta-analyses were not possible due to differing interventions and outcomes.

\section{Finger foods}

Two CCTs [59, 60] assessed use of finger foods. One [59] evaluated six months of finger food menu for 12 cognitively impaired residents with poor intake and limited use of cutlery, finding weight-loss arrested in 10/12 participants and eating independence improved (though no numbers or statistical analysis were provided). The other [60] assessed effects of increased finger food provision on weight and food consumption of 43 US AD care centre residents. The intervention, though aiming to increase numbers of finger foods provided, had implementation problems in that numbers of finger foods provided increased minimally (2.57 to 2.71 [breakfast]; 2.19 to 2.38 [lunch] and 2.0 to 3.8 [dinner]) over the six month study period. Body weight did not increase, though the proportion of food eaten increased by $3 \%(p<0.05)$.

\section{Other food modification}

Two RCTs [61, 62] assessed effects of food modification. Three months meal replacement with lyophilised (freezedried) foods and advice to residents and carers vs advice alone was investigated in 53 people with severe cognitive impairment [61], reporting improved weight $(+2.06 \mathrm{~kg}$ vs $+0.32 \mathrm{~kg}, p<0.05)$ and serum albumin
(3.76 mg/dl vs $1.13 \mathrm{mg} / \mathrm{dl}, p<0.05$ ), but no differences in MNA, eating behaviour, cognition or mortality. A crossover RCT [62] assessed 21 days high-carbohydrate dinners compared to usual intake in 34 nursing home residents with $\mathrm{AD}$. Energy intake increased, but weight was not presented for the first part of the crossover, so could not be assessed.

\section{Food modification as part of multi-component interventions}

One RCT [31] (discussed under multicomponent ONS interventions) and three CCTs [33, 63, 64] assessed food modification as part of multi-component interventions: One CCT [33] found increased serum albumin (3.0 to $3.4 \mathrm{~g} / \mathrm{dL})$ at 18 months in malnourished people with severe dementia living on a dementia unit, without effects on weight or BMI; the second [63] reported significant weight gain after nine months of enhanced dietetic time, high-energy snacks, high-energy/protein mashed potatoes, high-protein milk and blended enhanced breakfasts ( $4.8 \%$ vs. $-4.5 \%, p<0.001)$ in orally fed people with dementia (dementia stage not stated); and the third [64] found that increased choice, ability to self-serve, improved atmosphere and readily available drinks and snacks had no effect on nutritional status, hydration or enjoyment of food in care home residents with low mean MMSE (not selected for cognitive or nutritional status).

\section{Effects of eating and drinking assistance}

Eating and drinking assistance was defined as direct physical assistance provided by carers, staff or volunteers to enable eating and/or drinking including use of aids and extra staff, but not verbal prompting. Six interventions [33, 50, 65-67] assessed effects of eating or drinking assistance, of which all were judged at high or unclear risk of selection and performance bias, while one was at low risk of detection bias, and none were at low risk of bias overall (Additional file 3: Figure S3.7). Meta-analysis was not possible due to differing interventions and outcomes. One RCT and one CCT investigated effects of eating assistance on nutritional status of people with dementia [66, 67]. A US study [66] randomised nursing home residents to individual assistance at mealtimes or between-meals vs usual care. Energy intake was higher with assistance $(p=0.03)$, but BMI and weight (though measured) were not reported at 24 weeks. Staff time at 48 weeks was $>42$ minutes $/$ meal $/$ resident, and 14 minutes/snack/resident, and was $<10$ minutes/ meal/resident in the control group at 24 weeks. A CCT [67] (period 3) used volunteers to assist patients during mealtimes, reporting increased BMI $\left(+0.4 \mathrm{~kg} / \mathrm{m}^{2}, p<0.04\right)$ and energy intake $(+44 \mathrm{Kcal}, p<0.001)$ after 12 weeks (though it is surprising that such small changes were statistically significant in seven participants). 


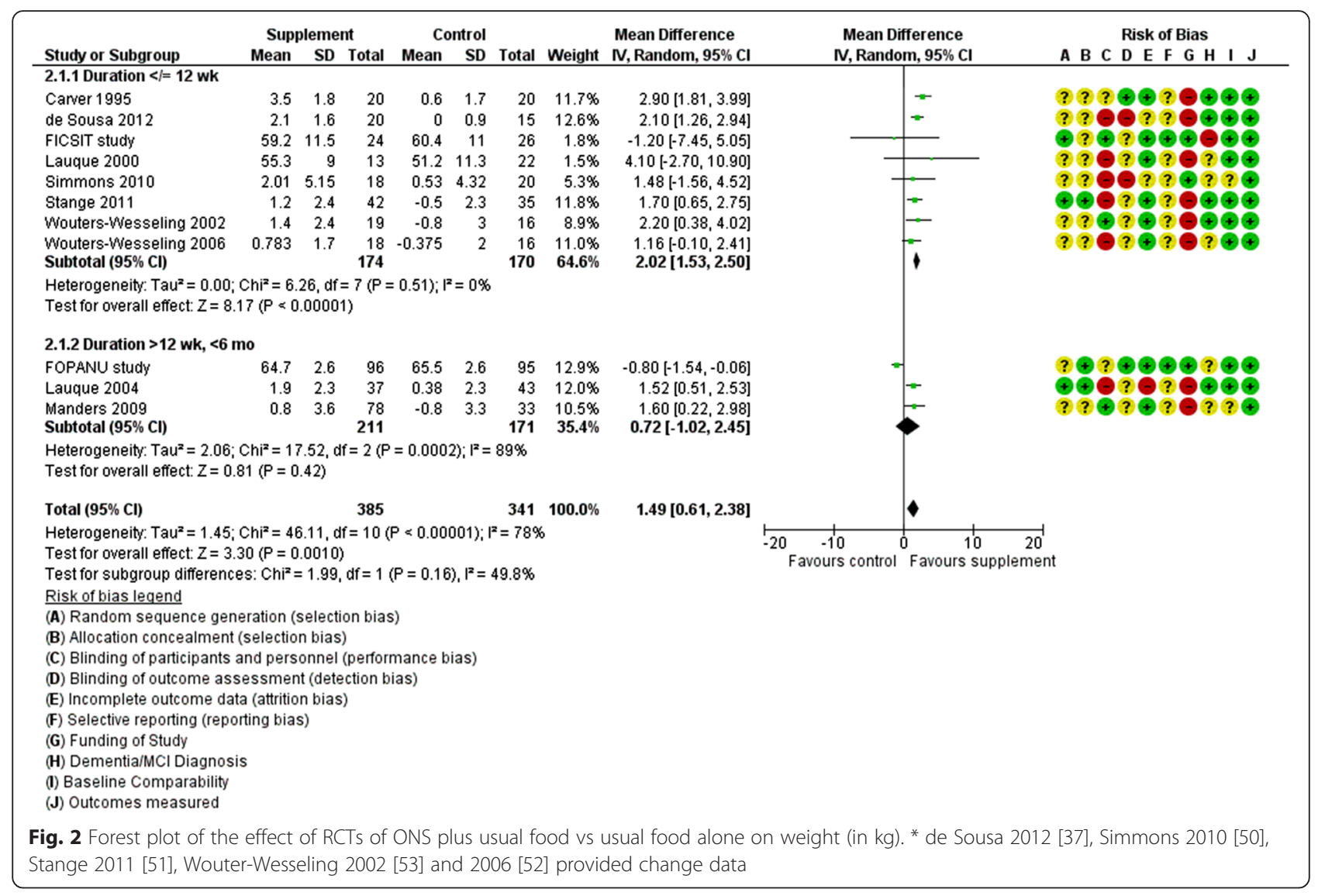

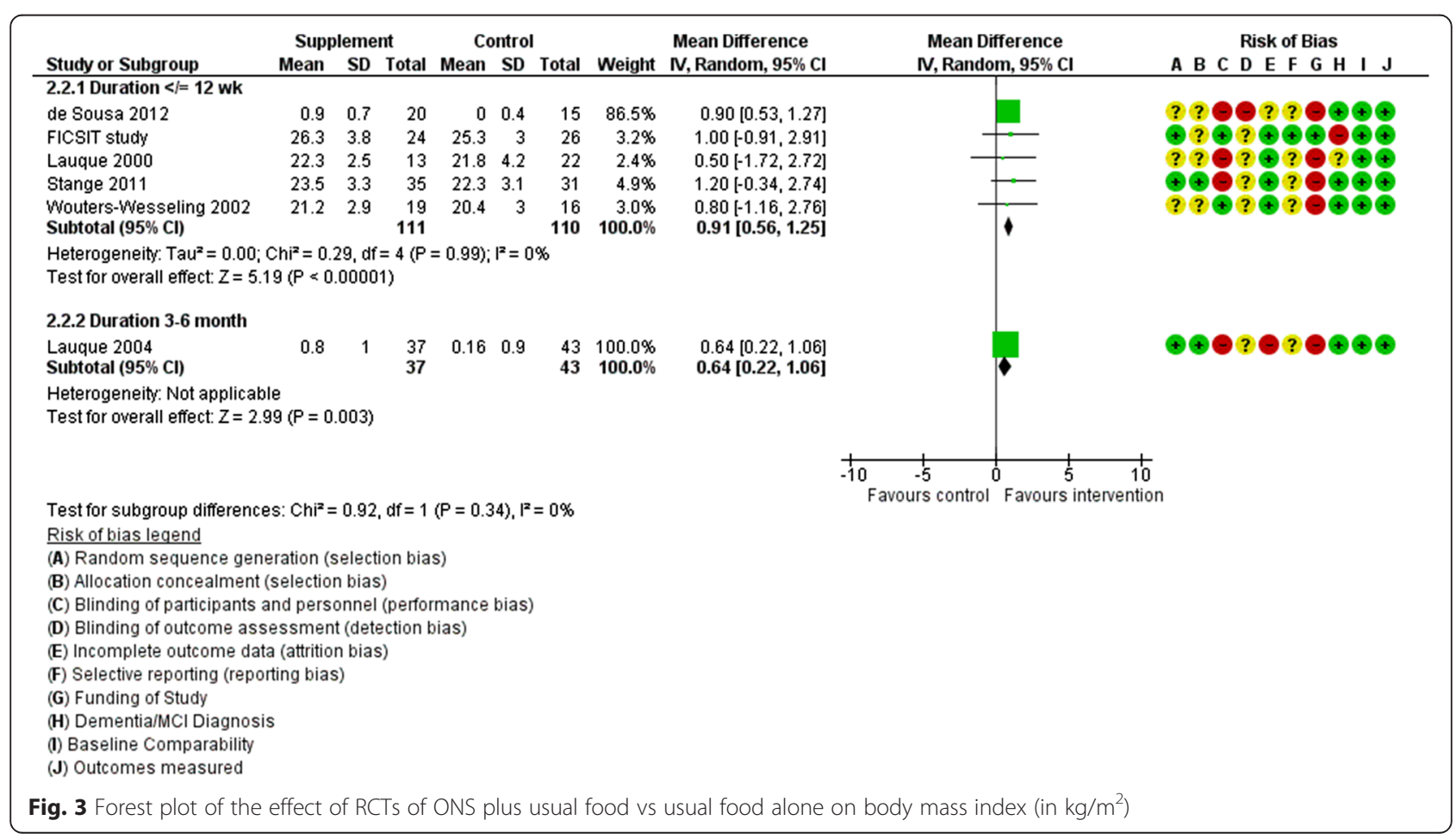


Four interventions $[33,50,65]$ had eating assistance as part of a multicomponent intervention: A CCT [33] (discussed above) included mealtime assistance, finding increased serum albumin at 18 months, without effects on weight or BMI; a complex 32-week CCT (including exercise, toileting and drinking prompts) [65] found no change in meal-time food or fluid intake, or hydration status; an RCT [50] provided assistance to enhance eating intake and independence alongside between-meal nutritional supplements or nutritious snacks compared to usual care, finding no effect on weight or energy intake at five weeks, but with increased staff time and costs.

\section{Effects of interventions with a strong social element around eating and drinking}

Of four CCTs [68-71] which studied the promotion of eating and/or drinking alongside a social activity or within a social context, all were at high or unclear risk of selection and performance bias, and one was at low risk of detection bias (Additional file 3: Figure S3.8). None was at low risk of bias overall.

Family-style meals in five US moderate-severe dementia unit residents were associated with increased resident participation in mealtime tasks, appropriate communication, frequency of praise and carer satisfaction with residents' participation levels, but statistical significance was unclear [68]. Another study of family-style meals taken with staff [69] reported increased weight $(+3.4 \mathrm{~kg}$ vs $-2.2 \mathrm{~kg}, p=0.02)$ in 18 patients with severe $\mathrm{AD}$, while comments and observations suggested improvements in autonomy, quality of interactions and other aspects of enjoyment of meals by patients. A study of reminiscence cooking therapy in 12 Taiwanese mild-moderate dementia nursing home residents improved most personal interaction scale items including feeling of happiness $(p=0.01)$ and positive communication $(p=0.05)$ [70]. A facilitated breakfast club compared to discussion with coffee in 40 people with mild-moderate AD in a dementia unit [71] reported significantly higher scores for interest, involvement and procedural memory, significantly better functional and cognition status.

\section{Answers to questions from lay stakeholders}

We found limited data to answer the questions formulated through public involvement (Table 1).

\section{Discussion}

To our knowledge, this is the first systematic review to assess the effectiveness of a full range of direct interventions aiming to improve, maintain or facilitate eating and drinking in adults with dementia of any type, any degree and in any setting. The 43 interventions investigated the effect of oral supplements, food/drink modification (including their use for people with swallowing problems), eating assistance and social support. Most interventions occurred within residential institutions of various types, but three interventions on people with MCI were in the community [41-43], one a treatment centre (assumed to be an outpatient setting [72]), one in day centres [48] and one unclear [55]. Included studies were mostly small (from five to 515 participants, with only seven having $\geq 100$ participants $[31,35,46,47,58,64,73]$ and none were at low risk of bias.

Meta-analysis of RCTs of ONS suggest small, short term, statistically significant effects (of ONS with usual food compared to usual food alone) on weight, BMI, MNA and energy intake, but effects were too small to be clinically useful, and it is unclear whether these effects were sustained in the longer term (Figs. 2 and 3). There were no clear effects on the relevant and important outcomes quality of life, functional or cognitive status, hydration status or mortality, nor on fluid intake, and data on more useful nutritional outcomes (such as albumin or iron status markers) were rare. There was insufficient evidence to assess effects of fruit juice supplements, nutrients without additional energy and protein or snacks between meals.

We found limited evidence that, for those with swallowing problems, dysphagia diets improved some nutritional markers [57]. Evidence for the utility of cervical spine mobilisation [56], different consistencies of thickened drinks, and chin-down drinking [58], were less clear. Effects of food modification were also mixed. In those unable to use cutlery, there was little evidence of finger food utility, with one study showing problems in implementation [60] and the other suggesting positive effects but with no numerical data provided [59]. In those with severe dementia there was some evidence that liquid or semi-liquid foods improved weight a little over 3 months (but not eating behaviour) [61]. Multicomponent interventions including food modification increased weight and BMI in some cases $[31,63]$ but not others [33, 64].

Studies assessing effects of assistance with eating and drinking suggested little effect on nutritional outcomes. Studies assessing social interventions were small but consistently suggested the possibility of improving important aspects of quality of life including autonomy, communication, mood, involvement and participation in meaningful activity in the context of food and drink [68-71].

As this systematic review did not find sufficient high quality evidence to state that any particular intervention was clearly effective, or clearly ineffective, we suggest that these interventions require further research. High quality, well designed randomised trials should be tailored specifically for people with dementia at different stages of the disease and in different settings. They 
should include robust outcomes such as weight change to clarify nutritional status, serum osmolality to inform on hydration status and health outcomes (several studies reported improvements in intake, without accompanying improvements in nutritional status indicating that intake measures should be interpreted cautiously). Trials will ideally include input from people with dementia, and their formal and informal carers in study design. Trials should be powered to produce useful data and consider longer term effects (over at least 6 months) of interventions.

Despite no clear evidence of effectiveness, people with dementia and their carers regularly have to deal with problems around eating and drinking, including needing to be fed, food refusal, and swallowing difficulties [7]. For this reason, they may like to read about the different interventions that have been tested, and choose one or more of the interventions to help solve their own problems. The possible interventions are detailed in Table 4 .

While previous systematic reviews have assessed potential nutritional causes and prevention of dementia [74-76], literature examining the effectiveness of interventions to support people with dementia to eat and drink well is more limited. Systematic reviews exploring effectiveness of interventions to support eating and drinking in people with dementia were either limited to residential care settings [23-25], focused on limited interventions or outcomes [26], or carried out using limited or ill-described searches $[27,28]$, hence leaving evidence gaps.

\section{Strengths and limitations}

A strength of this systematic review is that after an extensive search we found a comprehensive set of interventions aiming to improve, maintain or facilitate eating and drinking in people with dementia and MCI. All study selection and data extraction was conducted in duplicate and we carried out meta-analyses where studies were appropriately similar in intervention, comparison and outcome, but this was only feasible for RCTs of ONS. The scope was enhanced by lay stakeholder input to ensure we investigated relevant objectives. A limitation of our review may be that despite the extensive search, some studies might have been missed due to poor indexing and abstracts omitting to identify participants as having dementia or cognitive impairment. To overcome this, we included studies where cognitive scores suggested that most participants were cognitively impaired even when dementia was not mentioned or formally diagnosed.

We assessed interventions for swallowing problems in people with cognitive limitations, and found very limited evidence of the utility of interventions. However, whilst swallowing problems are common in people with dementia it is likely that results of research on interventions for people with swallowing problems but without dementia are transferrable to people with dementia, so the evidence base is much larger. Recent systematic reviews on interventions for people with swallowing problems provide a fuller data set $[77,78]$.

Table 4 Promising interventions that are presently unproven, but that warrant early reassessment in high quality and well powered RCTs ${ }^{a}$

\begin{tabular}{|c|c|}
\hline Aim & Potential interventions (presently unproven) which warrant early reassessment \\
\hline Increase weight and/or BMI & $\begin{array}{l}\text { o Oral Nutrition Supplements (ONS) (Figs. } 2 \text { and 3) } \\
\text { o ONS, gratin diet for those with swallowing problems, plus exercise and oral care (Beck) [31] } \\
\text { o Dysphagia diet (reformed minced and pureed foods and thickened fluids) for those with } \\
\text { swallowing problems (Germain) [57] } \\
\text { o Meal replacement with commercial lyophilised supplement (Salas-Salvado) [61] } \\
\text { o Multifactorial intervention including enhanced menu, individualised food service, more } \\
\text { dietetic time, increased nutritional awareness and communication (Keller) [63] } \\
\text { o Individual mealtime assistance (Wong) [67] } \\
\text { o Shared mealtime with staff (Charras) [69] }\end{array}$ \\
\hline Improve hydration & $\begin{array}{l}\text { o No particularly useful interventions were noted, but cervical spine manipulation } \\
\text { appeared to increase dysphagia limit for those with swallowing problems (Bautmans) [56] }\end{array}$ \\
\hline $\begin{array}{l}\text { Support meaningful engagement with food } \\
\text { and/or drink }\end{array}$ & $\begin{array}{l}\text { o Eating with carers (Charras) [69] } \\
\text { o Family style meals for people with dementia, enhanced further by staff training (Altus) [68] } \\
\text { o Facilitated breakfast club with supported involvement } \\
\text { in preparing, conversing, eating and clearing up (Santo Pietro) [71] }\end{array}$ \\
\hline Improve quality of life & o Reminiscence cooking sessions (Huang 2009) [70] \\
\hline Support eating independence & o No particularly useful interventions assessed \\
\hline $\begin{array}{l}\text { Improve quantity, quality or adequacy of food } \\
\text { or fluid intake }\end{array}$ & $\begin{array}{l}\text { O Combination of ONS, gratin diet, exercise and oral care (Beck) [32]o Finger food provision } \\
\text { (Soltesz) [60] } \\
\text { o High carbohydrate dinners (Young) [62] } \\
\text { o Meal time or between meal feeding } \\
\text { assistance, or individual mealtime assistance (Simmons 2008, Wong 2008) [66, 67] } \\
\text { o Dysphagia diet (reformed minced and pureed foods and thickened fluids) for those with } \\
\text { swallowing problems (Germain) [57] }\end{array}$ \\
\hline
\end{tabular}

alf you or someone you care for is experiencing difficulties with eating or drinking ALWAYS discuss these eating and drinking problems with your/their doctor, and ask to be referred to a dietitian and/or Speech and Language Therapist 
As included studies often lacked details of dementia diagnosis, type and/or staging it was difficult to assess what interventions may be useful in different types and stages of dementia. There is also a lack of clear data on nutritional and hydration status of participants, and studies not choosing people with specific nutritional deficits may limit our ability to see effects of interventions on nutritional outcomes [79]. Eating and drinking problems differ at different dementia stages, so it is likely that interventions will be stage- or problem-specific. Positive effects of nutritional supplements have been reported by other reviews $[80,81]$ but these reviews did not consider duration. Previous reviews have not considered meaningful activity or quality of life as primary outcomes, despite significantly positive relationships between psychological wellbeing and nutritional status in dementia [80-82]. Outcomes such as satisfaction, autonomy and quality of life have been highlighted in an expert-led research agenda as important determinants of interventions to improve food and fluid intake for older people, but were reported in few included studies [83]. The wide range of interventions and variable outcome measures reported make it difficult to compare results between studies.

Although we could not report definitive evidence on effectiveness of one or more interventions, by reviewing all available studies we have presented evidence on potentially useful interventions that could (and should) be considered for further research. We present a list of potentially useful interventions that need to be assessed in high quality and well powered RCTs to properly assess their efficacy (Table 4), and this may be a useful source of inspiration for people with dementia and their carers as suggestions to try out in dealing with specific eating and drinking problems, even though they have not been shown to be clearly effective. However, it should be noted that potentially effective interventions may not have appeared effective in this systematic review because many of the studies were underpowered, and so unable to suggest statistically significant benefits or harms.

Our finding that strong social support around food and drink are positive for quality of life is supported by qualitative research in people with dementia that "eating together reveals the essence of what it is to be human" and mealtimes reflect identity and connections [84] and anthropological research suggesting that eating together is a core human activity important to building social groups [85]. This is a priority for the research agenda.

\section{Conclusions}

While we found no definitive evidence on effectiveness, or lack of effectiveness, of specific interventions, studies were small and short term. As people with cognitive impairment and their carers have to tackle eating problems despite this lack of evidence, promising interventions have been listed and include: Oral nutrition supplements; pureed and reformed foods; thickened fluids; individual mealtime or between-meal assistance; family style meals and meals shared with staff or carers; meals with a facilitated social element; reminiscence cooking; finger food provision. The need remains for high quality trials tailored for people with progressive cognitive impairment and their carers assessing robust and relevant outcomes.

\section{Additional files}

Additional file 1: Full systematic review methodology for EDWINA (Eating and Drinking Well IN dementiA) systematic review. (DOCX $31 \mathrm{~kb}$ )

Additional file 2: Detailed characteristics of direct intervention EDWINA (Eating and Drinking Well IN dementiA) included studies. (DOCX $125 \mathrm{~kb}$ )

Additional file 3: Summary of study validity and further meta-analysis results of direct interventions. (DOCX $136 \mathrm{~kb}$ )

\section{Abbreviations}

CCT: non-randomised controlled trial; EDWINA: Eating and Drinking Well IN dementiA study; $\mathrm{MCl}$ : mild cognitive impairment; ONS: oral nutrition supplements; RCT: randomised controlled trial.

\section{Competing interests}

No authors declare a conflict of interest, except that one of the review authors is an author of one of the included studies in this systematic review. This study was assessed for inclusion, data extracted and assessed for validity by other reviewers, without reference to the paper's author.

\section{Authors' contributions}

The review idea was conceived by LH, FP and CF. AA managed the review. $A A$ and $L H$ drafted the protocol with expert and stakeholder input from $M C$, VC, AD, AH, AK, JP, KR, DS and CF. AA and LH designed the searches and AA ran the searches. AA and $\mathrm{LH}$ screened studies for inclusion/exclusion. AA extracted data and assessed study quality. DB, AD, CF, LG, AK, FL, JL, KR, and $\mathrm{LH}$ duplicated the data extraction and quality assessment. $\mathrm{AA}, \mathrm{DB}$ and $\mathrm{LH}$ conducted the analysis and drafted the review. All authors commented on and approved the final draft.

\section{Acknowledgments}

We are grateful to all our lay stakeholders who contributed questions, thoughts and ideas to the systematic review protocol. We are also grateful to authors Dr Mary Coyne and Dr Patricia Heyn for providing further data/ publications on their studies. This article summarises independent research funded in part by the National Institute for Health Research (NIHR),

Collaboration for Leadership in Applied Health Research \& Care, East of England (CLAHRC EOE), and in part by the National Institute of Health Research Fellowship programme (NIHR-CDF-2011-04-025). The views expressed are those of the authors and not necessarily those of the NHS, the NIHR or the Department of Health. The funders had no part in the collection, analysis or interpretation of data; in the writing of the report; and in the decision to submit the article for publication.

\section{Author details}

${ }^{1}$ Norwich Medical School, University of East Anglia, Norwich Research Park, Norfolk NR4 7TJ, UK. ${ }^{2}$ Age UK Norfolk, 300 St Faith's Road, Old Catton, Norwich NR6 7BJ, UK. ${ }^{3}$ NorseCare, Lancaster House 16 Central Avenue St Andrew's Business Park, Norwich NR7 OHR, UK. ${ }^{4}$ School of Health and Social Work, University of Hertfordshire, Hatfield, Hertfordshire AL10 9AB, UK. ${ }^{5}$ School of Health Sciences, University of East Anglia, Norwich Research Park, Norfolk NR4 7TJ, UK. ' Norfolk and Norwich University Hospital, Colney Lane, Norwich NR4 7UY, UK. 'King's College Hospital NHS Foundation Trust, Denmark Hill, London SE5 9RS, UK. ${ }^{8}$ Norfolk and Suffolk NHS Foundation 
Trust, Hellesdon Hospital, Drayton High Road, Norwich NR6 5BE, UK. ${ }^{9}$ Present address: Royal College of Paediatrics and Child Health, 5-11 Theobalds Road, London WC1X 8SH, UK.

Received: 27 September 2015 Accepted: 12 January 2016

Published: 22 January 2016

\section{References}

1. Abdelhamid A, Bunn D, Dickinson A, Killett A, Poland F, Potter J et al.: Effectiveness of interventions to improve, maintain or faciltate oral food and/or drink intake in people with dementia. PROSPERO 2014 CRD42014007611: http://www.crd.york.ac.uk/PROSPERO/display_record. asp?!D=CRD42014007611 .

2. World Health Organization: Dementia: Fact sheet N No362. accessed 2508 2015: http://www.who.int/mediacentre/factsheets/fs362/en/; 2015.

3. Alzheimer's Disease International: World Alzheimer Report 2009: The Global Prevalence of Dementia. accessed 2508 2015: http://www.alz.co.uk/ research/world-report-2009; 2015 .

4. Prince M, Bryce R, Albanese E, Wimo A, Ribeiro W, Ferri CP. The global prevalence of dementia: a systematic review and metaanalysis. Alzheimers Dement. 2013;9:63-75.

5. Corrada MM, Brookmeyer R, Paganini AM, Berlau D, Kawas CH. Dementia Incidence Continues to Increase with Age in the Oldest Old The 90+ Study. Ann Neurol. 2010;67:114-21.

6. Matthews FE, Arthur A, Barnes LE, Bond J, Jagger C, Robinson L, et al. A two-decade comparison of prevalence of dementia in individuals aged 65 years and older from three geographical areas of England: results of the Cognitive Function and Ageing Study I and II. Lancet. 2013;302:105-12.

7. Volicer L, Seltzer B, Rheaume Y, Karner J, Glennon M, Riley ME, et al. Eating Difficulties in Patients With Probable Dementia of the Alzheimer Type. J Geriatr Psychiatry Neurol. 1989;2:188-95.

8. Alzheimer's Society, James Lind Alliance. Outcomes of the James Lind Alliance Dementia priority setting partnership. 2013. http://alzheimers.org.uk/site/scripts/ documents_info.php?documentID=1804, accessed 2508 2015, Alzheimer's Society.

9. Morley JE, Caplan G, Cesari M, Dong B, Flaherty JH, Grossberg GT, et al. International survey of nursing home research priorities. J Am Med Dir Assoc. 2014;15:309-12.

10. Camina Martin MA, Barrera Ortega S, Dominguez Rodriguez L, Couceiro Muino C, de Mateo SB, del Rio MP R. Presence of malnutrition and risk of malnutrition in institutionalized elderly with dementia according to the type and deterioration stage. Nutr Hosp. 2012;27:434-40

11. Haveman-Nies A, de Groot LC, Van Staveren WA. Fluid intake of elderly Europeans. The Journal of Nutrition, Health and Aging. 1997;1:151-5.

12. Jesus P, Desport JC, Massoulard A, Villemonteix C, Baptiste A, Gindre-Poulvelarie L, et al. Nutritional assessment and follow-up of residents with and without dementia in nursing homes in the Limousin region of France: a health network initiative. J Nutr Health Aging. 2012;16:504-8.

13. Siervo M, Bunn D, Prado C, Hooper L. Accuracy of prediction equations for serum osmolarity in frail older people with and without diabetes. Am J Clin Nutr. 2014;100:867-76.

14. Natalwala A, Potluri R, Uppal H, Heun R. Reasons for hospital admissions in dementia patients in Birmingham, UK, during 2002-2007. Dement Geriatr Cogn Disord. 2008;26:499-505.

15. Hooper L, Bunn D, Jimoh FO, Fairweather-Tait SJ. Water-loss dehydration and aging. Mech Ageing Dev. 2014;136-7:50-8.

16. Feehally J, Khosravi M. Effects of acute and chronic hypohydration on kidney health and function. Nutr Rev. 2015;73:110-9.

17. Elmadfa I, Meyer AL. Patterns of drinking and eating across the European Union: implications for hydration status. Nutr Rev. 2015;73:141-7.

18. Abdelhamid A, Bunn DK, Copley M, Cowap V, Dickinson A, Howe A, et al. Indirect interventions to for improving, maintaining or facilitating oral food and/or drink intake in people with dementia: a systematic review. Submitted. 2015

19. Alzheimer's Disease International: World Alzheimer Report 2013: An analysis of long-term care for dementia. accessed 2508 2015: http://www.alz.co.uk/ research/world-report-2013; 2013.

20. Higgins JPT, Green S. Cochrane Handbook for Systematic Reviews of Interventions Version 5.1.0 [updated March 2011]. http://handbook. cochrane.org/. 2011. The Cochrane Collaboration. 25-8-2015.
21. Moher D, Liberati A, Tetzlaff J, Altman DG, The PRISMA Group. Preferred Reporting Items for Systematic Reviews and Meta-Analyses: The PRISMA Statement. PLoS Med. 2009;6, e1000097.

22. Hooper L, Abdelhamid A, Atreed NJ, Campbell WW, Chassagne P, Channell AM, et al. Clinical symptoms, signs and tests for identification of impending and current water-loss dehydration in older people. Cochrane Database Syst Rev. 2015;2015, CD009647.

23. Hines S, McCrow J, Abbey J, Gledhill S. Thickened fluids for people with dementia in residential aged care facilities: a comprehensive systematic review. JBI Database of Systematic Reviews and Implementation Reports. 2009;7:761-824.

24. Hines S, Wilson J, McCrow J, Abbey J, Sacre S. Oral liquid nutritional supplements for people with dementia in residential aged care facilities: a systematic review. JBI Database of Systematic Reviews and Implementation Reports. 2010;8:1-52.

25. Jackson J, Currie K, Graham C, Robb Y. The effectiveness of interventions to reduce undernutrition and promote eating in older adults with dementia: A systematic review. JBI Database of Systematic Reviews and Implementation Reports. 2011;9:1509-50.

26. Whear R, Abbott R, Thompson-Coon J, Bethel A, Rogers M, Hemsley A, et al. Effectiveness of mealtime interventions on behavior symptoms of people with dementia living in care homes: a systematic review. J Am Med Dir Assoc. 2014;15:185-93.

27. Alagiakrishnan K, Bhanji RA, Kurian M. Evaluation and management of oropharyngeal dysphagia in different types of dementia: a systematic review. Arch Gerontol Geriatr. 2013;56:1-9.

28. Hanson LC, Ersek M, Gilliam R, Carey TS. Oral feeding options for people with dementia: a systematic review. J Am Geriatr Soc. 2011;59:463-72.

29. Higgins JPT, Altman DG, Gotzsche PC, Juni P, Moher D, Oxman AD, et al. The Cochrane Collaboration's tool for assessing risk of bias in randomised trials. Br Med J. 2011;343:d5928

30. Abalan F, Manciet G, Dartiques JF, Decamps A, Zapata E, Saumtally B, et al. Nutrition and SDAT. Biol Psychiatry. 1992;31:103-5.

31. Beck AM, Damkjaer K, Sørbye LW. Physical and social functional abilities seem to be maintained by a multifaceted randomized controlled nutritional intervention among old (>65 years) Danish nursing home residents. Arch Gerontol Geriatr. 2010;50:351-5.

32. Beck AM, Ovesen L, Schroll M. Home-made oral supplement as nutritional support of old nursing home residents, who are undernourished or at risk of undernutrition based on the MNA. A pilot trial. Aging Clin Exp Res. 2002;14:212-5.

33. Boffelli S, Rozzini R, Trabucci M. Nutritional Intervention in Special Care Units for Dementia. J Am Geriatr Soc. 2004;52:1216-7.

34. Carlsson M, Gustafson $Y$, Haglin L, Eriksson S. The feasibility of serving liquid yoghurt supplemented with probiotic bacteria, Lactobacillus rhamnosus LB 21, and Lactococcus lactis L1A - A pilot study among old people with dementia in a residential care facility. J Nutr Health Aging. 2009;13:813-9.

35. Carlsson M, Littbrand H, Gustafson Y, Lundin-Olsson L, Lindelof N, Rosendahl E, et al. Effects of high-intensity exercise and protein supplement on muscle mass in ADL dependent older people with and without malnutrition: a randomized controlled trial. J Nutr Health Aging. 2011;15:554-60.

36. Carver AD, Dobson AM. Effects of dietary supplementation of elderly demented hospital residents. J Hum Nutr Diet. 1995;8:389-94.

37. de Sousa OL, Amaral TF. Three-week nutritional supplementation effect on long-term nutritional status of patients with mild Alzheimer Disease. Alzheimer Dis Assoc Disord. 2012;26:119-23.

38. Faxen-Irving G, Andren-Olsson B, af Geijerstam A, Basun H, Cederholm T. The effect of nutritional intervention in elderly subjects residing in groupliving for the demented. Eur J Clin Nutr. 2002;56:221-7.

39. Fiatarone Singh MA, Bernstein MA, Ryan AD, O'Neill EF, Clements KM, Evans WJ. The effect of oral nutritional supplements on habitual dietary quality and quantity in frail elders. J Nutr Health Aging. 2000;4:5-12.

40. Gregorio PG, Diaz SPR, Casado JMR. Dementia and Nutrition. Intervention study in institutionalized patients with Alzheimer Disease. J Nutr Health Aging. 2003;7:304-8.

41. Krikorian R, Boespflug EL, Fleck DE, Stein AL, Wightman JD, Shidler MD, et al. Concord grape juice supplementation and neurocognitive function in human aging. J Agric Food Chem. 2012;60:5736-42.

42. Krikorian R, Nash TA, Shidler MD, Shukitt-Hale B, Joseph JA. Concord grape juice supplementation improves memory function in older adults with mild cognitive impairment. Br J Nutr. 2010;103:730-4. 
43. Krikorian R, Shidler MD, Nash TA, Kalt W, Vinqvist-Tymchuk MR, Shukitt-Hale B, et al. Blueberry supplementation improves memory in older adults. J Agric Food Chem. 2010;58:3996-4000.

44. Lauque S, Arnaud-Battandier F, Mansourian R, Guigoz Y, Paintin M, Nourhashemi F, et al. Protein-energy oral supplementation in malnourished nursing-home residents. A controlled trial. Age Ageing. 2000;29:51-6.

45. Lauque S, Arnaud-Battandier F, Gillette S, Plaze JM, Andrieu S, Cantet C, et al. Improvement of weight and fat-free mass with oral nutritional supplementation in patients with Alzheimer's disease at risk of malnutrition: a prospective randomized study. J Am Geriatr Soc. 2004;52:1702-7.

46. Manders M, De Groot LCPG, Hoefnagels WHL, Dhonukshe-Rutten RAM, Wouters-Wesseling W, Mulders AJMJ, et al. The effect of a nutrient dense drink on mental and physical function in institutionalized elderly people. J Nutr Health Aging. 2009;13:760-7.

47. Navratilova M, Jarkovsky J, Ceskova E, Leonard B, Sobotka L. Alzheimer disease: Malnutrition and nutritional support. Clin Exp Pharmacol Physiol. 2007;34:511-3.

48. Planas M, Conde M, Audivert S, Perez-Portabella C, Burgos R, Chacon P, et al. Micronutrient supplementation in mild Alzheimer disease patients. Clin Nutr. 2004;23:265-72.

49. Scheltens P, Kamphuis PJ, Verhey FR, Olde Rikkert MG, Wurtman RJ, Wilkinson D, et al. Efficacy of a medical food in mild Alzheimer's disease: A randomized, controlled trial. Alzheimers Dement. 2010;6:1-10.

50. Simmons S, Zhuo X, Keeler E. Cost-effectiveness of nutrition interventions in nursing home residents: A pilot intervention. J Nutr Health Aging. 2010;14:367-72.

51. Stange I, Bartram M, Liao Y, Poeschl K, Kolpatzik S, Uter W: Effects of a lowvolume, nutrient- and energy-dense oral nutritional supplement on nutritional and functional status: A randomized, controlled trial in nursing home residents. J Am Med Dir Assoc 2013, 14: 628 e1-628 e8.

52. Wouters-Wesseling W, Slump E, Kleijer CN, de Groot LC, Van Staveren WA. Early nutritional supplementation immediately after diagnosis of infectious disease improves body weight in psychogeriatric nursing home residents. Aging Clin Exp Res. 2006;18:70-4.

53. Wouters-Wesseling W, Wouters AE, Kleijer CN, Bindels JG, Groot CP, Staveren WA. Study of the effect of a liquid nutrition supplement on the nutritional status of psycho-geriatric nursing home patients. Eur J Clin Nutr. 2002:56:245-51

54. Young KW, Greenwood CE, van Reekum R, Binns MA. Providing nutrition supplements to institutionalized seniors with probable Alzheimer's disease is least beneficial to those with low body weight status. J Am Geriatr Soc. 2004:52:1305-12.

55. Pivi GA, da Silva RV, Juliano Y, Novo NF, Okamoto $\|$, Brant CQ, et al. A prospective study of nutrition education and oral nutritional supplementation in patients with Alzheimer's disease. Nutr J. 2011;10:98.

56. Bautmans I, Demarteau J, Cruts B, Lemper JC, Mets T. Dysphagia in elderly nursing home residents with severe cognitive impairment can be attenuated by cervical spine mobilization. J Rehabil Med. 2008;40:755-60.

57. Germain I, Dufresne T, Gray-Donald K. A Novel Dysphagia Diet Improves the Nutrient Intake of Institutionalized Elders. J Am Diet Assoc. 2006;106:1614-23.

58. Robbins J, Gensler G, Hind J, Logemann JA, Lindblad AS, Brandt D, et al. Comparison of 2 interventions for liquid aspiration on pneumonia incidence: A randomized trial. Ann Intern Med. 2008;148:509-18.

59. Jean LA. "Finger food menu" restores independence in dining. Health Care Food Nutr Focus. 1997:14:4-6.

60. Soltesz KS, Dayton JH. The effects of menu modification to increase dietary intake and maintain the weight of Alzheimer residents. Am J Alzheimers Dis Other Demen. 1995;10:20-3.

61. Salas-Salvado J, Torres M, Planas M, Altimir S, Pagan C, Gonzalez ME, et al. Effect of oral administration of a whole formula diet on nutritional and cognitive status in patients with Alzheimer's disease. Clin Nutr. 2005;24:390-7.

62. Young KW, Greenwood CE, Reekum R, Binns MA. A randomized, crossover trial of high-carbohydrate foods in nursing home residents with Alzheimer's disease: associations among intervention response, body mass index, and behavioral and cognitive function. J Gerontol A Biol Sci Med Sci. 2005;60:1039-45.

63. Keller HH, Gibbs AJ, Boudreau LD, Goy RE, Pattillo MS, Brown HM. Prevention of Weight Loss in Dementia with Comprehensive Nutritional Treatment. J Am Geriatr Soc. 2003;51:945-51.

64. Kenkmann A, Price GM, Bolton J, Hooper L. Health, wellbeing and nutritional status of older people living in UK care homes: an exploratory evaluation of changes in food and drink provision. BMC Geriatr. 2010;10:28.
65. Simmons SF, Alessi C, Schnelle JF. An intervention to increase fluid intake in nursing home residents: prompting and preference compliance. J Am Geriatr Soc. 2001;49:926-33.

66. Simmons SF, Keeler E, Zhuo X, Hickey KA, Sato HW, Schnelle JF. Prevention of unintentional weight loss in nursing home residents: a controlled trial of feeding assistance. J Am Geriatr Soc. 2008;56:1466-73.

67. Wong A, Burford S, Wyles CL, Mundy H, Sainsbury R. Evaluation of strategies to improve nutrition in people with dementia in an assessment unit. J Nutr Health Aging. 2008;12:309-12.

68. Altus DE, Engelman KK, Mathews RM. Using family-style meals to increase participation and communication in persons with dementia. J Gerontol Nurs. 2002;28:47-53.

69. Charras K, Fremontier M. Sharing meals with institutionalized people with dementia: a natural experiment. J Gerontol Soc Work. 2010;53:436-48.

70. Huang SL, Li CM, Yang CY, Chen JJ. Application of reminiscence treatment on older people with dementia: a case study in Pingtung, Taiwan. Journal of Nursing Research. 2009;17:112-9.

71. Santo Pietro MJ, Boczko F. The Breakfast Club: Results of a study examining the effectiveness of a multi-modality group communication treatment. Am J Alzheimers Dis Other Demen. 1998;13:146-58.

72. Scheltens P, Twisk JW, Blesa R, Scarpini E, Arnim CA, Bongers A, et al. Efficacy of Souvenaid in mild Alzheimer's disease: results from a randomized, controlled trial. J Alzheimers Dis. 2012;31:225-36.

73. Scheltens P, Verhey FRJ, Rikkert MGMO, Kamphuis PJGH, Wilkinson D, Kurz A. The efficacy of a medical food (Souvenaid) in Alzheimer's disease: Results from the first trial and design of future trials. Alzheimers Dement. 2009:5:258-9.

74. Scholey A, Owen L. Effects of chocolate on cognitive function and mood: a systematic review. Nutr Rev. 2013;71:665-81.

75. Ilomaki J, Jokanovic N, Tan ECK, Lonnroos E. Alcohol consumption, dementia and cognitive decline: an overview of systematic reviews. Curr Clin Pharmacol. 2015;10:204-12.

76. Panza F, Solfrizzi V, Burulli MR, Bonfiglio C, Guerra V, Osella A, et al. Coffee, tea and caffeine consumption and prevention of late-life congitive decline and dementia: a systematic review. J Nutr Health Aging. 2015;19:313-28.

77. Ashford J, McCabe D, Wheeler-Hegland K, Frymark T, Mullen R, Musson N, et al. Evidence-based systematic review: Oropharyngeal dysphagia behavioral treatments. Part III-Impact of dysphagia treatments on populations with neurological disorders. Journal of Rehabilitation Research and Development. 2009;46:195-204.

78. Steele CM, Abdulrahman WA, Ayanikalath S, arbon CEA, hen J, ichero JAY, et al. The influence of food texture and liquid consistency modification on swallowing physiology and function: a systematic review. Dysphagia. 2015:30:2-26.

79. Barnes JL, Tian M, Edens NK, Morris MK. Consideration of nutrient levels in studies of cognitive decline. Nutr Rev. 2014;72:707-19.

80. Allen VJ, Methven L, Gosney MA. Use of nutritional complete supplements in older adults with dementia: systematic review and meta-analysis of clinical outcomes. Clin Nutr. 2013;32:950-7.

81. Liu W, Cheon J, Thomas SA. Interventions on mealtime difficulties in older adults with dementia: a systematic review. Int J Nurs Stud. 2014;51:14-27.

82. Muurinen S, Savikko N, Soini H, Suominen M, Pitkala K. Nutrition and psychological well-being among long-term care residents with dementia. J Nutr Health Aging. 2015;19:178-82.

83. Keller $\mathrm{H}$, Beck AM, Namasivayam A. Improving food and fluid intake for older adults living in long-term care: a research agenda. J Am Med Dir Assoc. 2015;16:93-100.

84. Keller HH, Martin LS, Dupuis S, Genoe R, Cassolato C. Mealtimes and being connected in the community-based dementia context. Dementia. 2010;9: $191-213$.

85. Jones M. Feast: why humans share food. Oxford, England: Oxford University Press; 2007.

86. Fiatarone MA, O'Neill EF, Ryan ND, Clements KM, Solares GR, Nelson ME, et al. Exercise training and nutritional supplementation for physical frailty in very elderly people. N Engl J Med. 1994;330:1769-75.

87. Ory MG, Schechtman KB, Miller JP, Hadley EC, Fiatarone MA, Province MA, et al. Frailty and injuries in later life: the FICSIT trials. J Am Geriatr Soc. 1993;41:283-96

88. Fiatarone MA, O'Neill EF, Doyle N, Clements KM, Roberts SB, Kehayias JJ, et al. The Boston FICSIT Study: The effects of resistance training and nutritional 
supplementation on physical frailty in the oldest old. J Am Geriatr Soc. 1993:41:333-7.

89. Littbrand H, Carlsson M, Lundin-Olsson L, Lindelof N, Haglin L, Gustafson Y, et al. Effect of a high-intensity functional exercise program on functional balance: Preplanned subgroup analyses of a randomized controlled trial in residential care facilities. J Am Geriatr Soc. 2011;59:1274-82.

90. Rosendahl E, Lindelöf $\mathrm{N}$, Littbrand $\mathrm{H}$, Yifter-Lindgren $\mathrm{E}$, Lundin-Olsson L, Håglin L, et al. High-intensity functional exercise program and proteinenriched energy supplement for older persons dependent in activities of daily living: a randomised controlled trial. Aust J Physiother. 2006;52:105-13.

91. Scheltens P. Medical nutrition in Alzheimer's disease: First results and clinical trial. Eur J Pharmacol. 2011;668:e8-9.

92. Kamphuis PJ, Scheltens P, Hartmann T, Soininen H. The Souvenaid clinical trial program for Alzheimer's disease. Eur J Pharmacol. 2011;668, e30.

93. Kamphuis PJ, Verhey FR, Olde Rikkert MG, Twisk JW, Swinkels SH, Scheltens P. Efficacy of a medical food on cognition in Alzheimer's disease: results from secondary analyses of a randomized, controlled trial. J Nutr Health Aging. 2011;15:720-4.

94. Kamphuis P. The use of food for special medical purposes (product ID 4804/4805) in patients with early Alzheimer's disease. ISRCTN Register. http://www.controlled-trials.com/ISRCTN72254645. 2006. Series, Editor.

95. Scheltens P, Shah R, Bennett D, Wieggers RL, Hartmann T, Soininen H, et al. The Souvenaid(R) clinical trial program for Alzheimer's Disease [abstract]. Alzheimers Dement. 2012;8:602-3.

96. Scheltens P, Kamphuis PJ: Medical nutrition in Alzheimer's Disease: baseline characteristics of 3 Souvenaid(R) clinical studies [abstract]. Eur J Pharmacol 2011, 668: e31.

97. Stange I, Barthram M, Liao Y, Poschl K, Stehle P, Sieber CC et al.: Effects of oral nutritional supplements on functionality and quality of life of nursing home residents with malnutrition or its risk. Clin Nutr, Suppl 2011, 6 (1): 128

98. Stange I, Bartram M, Liao Y, Poeschl K, Stehle P, Sieber CC, et al. Impact of oral nutritional supplements on nutritional and functional status of nursing home residents with malnutrition or at risk. Eur Geriatr Med. 2011:2:S164.

99. Stange I, Bartram M, Liao Y, Poeschl K, Stehle P, Sieber CC, et al. Oral nutritional supplements and quality of life in nursing home residents with malnutrition or at risk. Eur Geriatr Med. 2011;2:S163-4.

100. Parrott MD, Young KW, Greenwood CE. Energy-containing nutritional supplements can affect usual energy intake postsupplementation in institutionalized seniors with probable Alzheimer's disease. J Am Geriatr Soc. 2006;54:1382-7.

101. Beck AM, Damkjaer K, Beyer N. Multifaceted nutritional intervention among nursing-home residents has a positive influence on nutrition and function. Nutrition. 2008;24:1073-80.

102. Logemann JA, Gensler G, Robbins J, Lindblad AS, Brandt D, Hind JA, et al. A randomized study of three interventions for aspiration of thin liquids in patients with dementia or Parkinson's disease. J Speech Lang Hear Res. 2008:51:173-83.

103. Hind JA, Gensler G, Brandt DK, Gardner PJ, Blumenthal L, Gramigna GD, et al. Comparison of trained clinician ratings with expert ratings of aspiration on videofluoroscopic images from a randomized clinical trial. Dysphagia. 2009;24:211-7.

104. Brandt DK, Hind JA, Robbins J, Lindblad AS, Gensler G, Gill G, et al. Challenges in the design and conduct of a randomized study of two interventions for liquid aspiration. Clinical Trials. 2006;3:457-68.

105. Keller HH, Gibbs-Ward A, Randall-Simpson J, Bocock MA, Dimou E. Meal rounds: an essential aspect of quality nutrition services in long-term care. J Am Med Dir Assoc. 2006;7:40-5.

106. Kenkmann A, Hooper L. The restaurant within the home: experiences of a restaurant-style dining provision in residential homes for older people. QAOA. 2012;13:98-110

107. Schnelle JF, Alessi C, Simmons SF, al-Samarrai N, Beck JC, Ouslander JG Translating clinical research into practice: a randomized controlled trial of exercise and continence care with nursing home residents. J Am Geriatr Soc. 2002;50:1476-83.

\section{Submit your next manuscript to BioMed Central and we will help you at every step:}

- We accept pre-submission inquiries

- Our selector tool helps you to find the most relevant journal

- We provide round the clock customer support

- Convenient online submission

- Thorough peer review

- Inclusion in PubMed and all major indexing services

- Maximum visibility for your research

Submit your manuscript at www.biomedcentral.com/submit

) Biomed Central 